\title{
Fast, Compact and Discriminative: Evaluation of Binary Descriptors for Mobile Applications
}

\author{
Simone Madeo and Miroslaw Bober, Member, IEEE
}

\begin{abstract}
Local feature descriptors underpin many diverse applications, supporting object recognition, image registration, database search, 3D reconstruction and more. The recent phenomenal growth in mobile devices and mobile computing in general has created demand for descriptors that are not only discriminative, but also compact in size and fast to extract and match. In response, a large number of binary descriptors have been proposed, each claiming to overcome some limitations of the predecessors. This paper provides a comprehensive evaluation of several promising binary designs. We show that existing evaluation methodologies are not sufficient to fully characterize descriptors' performance and propose a new evaluation protocol and a challenging dataset. In contrast to the previous reviews, we investigate the effects of the matching criteria, operating points and compaction methods, showing that they all have a major impact on the systems' design and performance. Finally, we provide descriptor extraction times for both general-purpose systems and mobile devices, in order to better understand the real complexity of the extraction task. The objective is to provide a comprehensive reference and a guide that will help in selection and design of the future descriptors.
\end{abstract}

Index Terms-Descriptor benchmarking, local feature descriptor, binary descriptor, image retrieval.

\section{INTRODUCTION}

$\mathbf{T}$ HE widespread use of mobile devices such as smartphones and tablets equipped with high-resolution cameras, power-constrained CPUs and limited storage led to the diffusion of new algorithms and communication paradigms for image and video applications [1]. The straightforward access to visual digital contents through pervasive networks also facilitates the development of many mobile applications based on large-scale image search and retrieval, e.g. e-commerce, retail catalogs, photo and art collections [2]. Such tasks deal with vast amounts of data: the processing is often partitioned between a resource-limited client and a powerful remote server, connected via a link with strict bandwidth constraints. The client usually processes a photo containing one or more target objects and transmits the extracted visual features to the server [3]-[6]. The query features are then compared to the features stored in a database in order to find the matches between objects. Depending on the application, the similarity score between database images and query image is computed and used to recognize an object or rank relevant images to be sent back to the client [7].

S. Madeo is with Real-Time Systems Laboratory (ReTiS Lab), Scuola Superiore Sant'Anna, Pisa, Italy and Centre of Vision, Speech and Signal Processing (CVSSP), University of Surrey, Guildford, UK (e-mail: s.madeo@sssup.it).

M. Bober is with Centre of Vision, Speech and Signal Processing (CVSSP), University of Surrey, Guildford, UK.

This research has received partial funding from the European Commission 7th Framework Programme under grant agreement Nr. 610691.

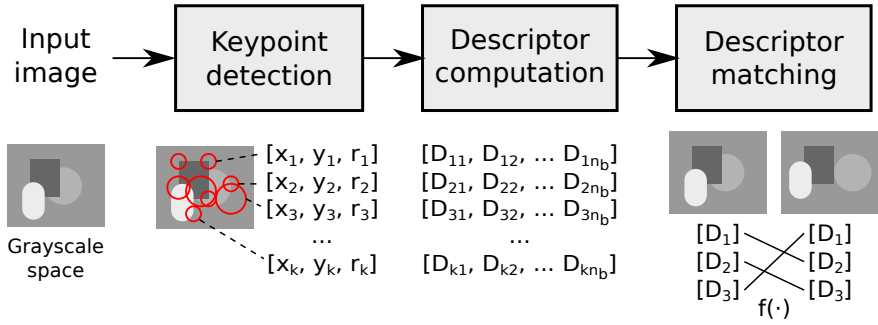

Fig. 1. Three-stage pipeline for feature extraction and matching. Keypoints are usually represented by their $(x, y)$ location and a parameter defining the size of the region to be encoded (radius $r$ in case of circles). Each region is used to encode a $n_{b}$-dimension descriptor $D_{i}, 0 \leq i<k$, where $k$ is the number of keypoints. Matching descriptors for two images are then selected by means of a distance function $f(\cdot)$ in order to assess their degree of similarity.

Visual features are usually coded in numerical vectors called descriptors, which robustly characterize the content of an image. Descriptors effectively reduce the computational complexity of the matching tasks on the server side and the amount of data to transmit over the network. According to the feature extraction procedure, descriptors are divided into two broad classes. Global descriptors represent the content of the image as a whole, typically using fixed-dimensional feature vectors which can be efficiently indexed and matched. They are well suited for large-scale applications by means of dedicated hashing algorithms [8], [9]. Nonetheless, the spatial layout of the scene is not directly addressed and the visual content is not locally encoded. Local descriptors deal with these limitations providing robust solutions to occlusions, significant geometric transformations (e.g. rotation, scale, translation, similarity) and color changes (e.g. luminance, contrast) [10]-[27].

Local features are typically extracted and compared using a three-stage pipeline (see Figure 1). First, a detector analyzes the image to extract a set of keypoints, i.e. salient points with a well-defined location representing the most informative parts of an image. For instance, blob detectors consider local extrema of the response of scale-invariant filters, usually designed to be approximations of the Laplacian of Gaussian (LoG) [28], [29], while corner detectors are based on 2D points having two dominant and different edge directions in their local neighborhood [6]. In the second stage, image patches around each keypoint are processed further and compactly represented with fixed-dimensional descriptors. Descriptors design must provide both discriminative power and transformations invariance. The importance of these properties is highlighted during the third stage, where the distance between descriptors is computed in order to find the best matches: the lower the distance, the more similar the correspondent patches.

As shown in Figure 1, the number of extracted keypoints 
is a critical parameter for the design of scalable systems and depends both on the image size and visual content. In order to reduce the complexity of the search procedure, keypoints may be grouped and jointly encoded exploiting mutual information and auxiliary feature space [30], [31]. The use of large visual codebooks typically accelerates the feature quantization and matching, however offline training and online processing require considerable computational resources and large amount of memory, therefore for large scale mobile image search codebook-free algorithms are preferred [32].

When feature extraction is performed on low-power devices, the choice of computationally efficient algorithms is essential. In 2010, while the state-of-the art was still largely defined by the first scale-invariant descriptor, SIFT [33], the first binary local descriptor, BRIEF [10], opened the floodgates to new designs. Binary descriptors are an attractive alternative for the following reasons:

- they dramatically reduce the matching time by using Hamming distance (XOR and bit count operations), which is natively supported on modern processors;

- their footprint is small, which means they can be loaded into memory for fast search or easily transmitted to the network;

- floating point operations are generally avoided or significantly reduced, ensuring a faster extraction time.

In this paper, we present an extensive comparative analysis of 21 promising binary descriptors characterizing local features. The major contributions can be summarized as follows:

- A full comparison evaluation on patch and image datasets with in-depth technical discussions on the reported results. In contrast to the previous reviews, the simultaneous effects of keypoint detectors, matching criteria, operating points and compaction methods are also investigated, showing that they all have a major impact on the system design and performance.

- An extension of the existing testing methodologies with a new evaluation protocol for image similarity able to improve the matching performance.

- A new metric able to summarize the overall performance based on several geometric, color and compression transformations.

- A study of existing operating points for ROC analysis and brute-force search for the optimum ratio test thresholds in order to fully characterize both distinctiveness and robustness of the descriptors.

- A new dataset reflecting typical challenges faced by mobile applications for objects retrieval, consisting of thousands of pictures extracted from video sequences shot using different mobile devices. Images are affected by several typical alterations such as different natural and artificial illuminations, shadows, reflections, motion blur, deformations, transparency effects and occlusions.

- A summary of descriptor extraction times, for both general-purpose systems and mobile devices, is also provided in order to better understand the real complexity of the extraction task.

The paper is structured as follows. Reviews of state-of-the- art binary descriptors and existing evaluation protocols and datasets are presented in Sections II and III respectively. Section IV introduces an evaluation framework with new matching paradigms. In Section $\mathrm{V}$ experimental results on previously published datasets are shown, and a new dataset for mobile applications is also proposed. Matching results are ranked by means of several quality metrics and descriptor extraction times. Finally, conclusions are presented in Section VI, with specific focus on selection and design of future descriptors.

\section{REVIEW OF STATE-OF-THE-ART BINARY DESCRIPTORS}

In this section we briefly introduce descriptors evaluated in our review, grouped by the approach employed to extract information from image patches.

1) Intensity-oriented descriptors: Descriptors in this class characterize an image patch $p$ of size $S \times S$ using a relatively small number $n_{b}$ of pairwise intensity comparisons $\phi$ :

$$
\phi\left(p ; x_{a}, x_{b}\right):= \begin{cases}1 & \text { if } p\left(x_{a}\right)<p\left(x_{b}\right) \\ 0 & \text { otherwise }\end{cases}
$$

where $p\left(x_{a}\right)$ and $p\left(x_{b}\right)$ are the pixel intensities at location $x_{a}$ and $x_{b}$. The $n_{b}$-dimensional bitstring $D^{\left(n_{b}\right)}$ is constructed by concatenating the bits corresponding to the results of individual comparisons based on a set of $n_{b}$ location pairs. Since intensity-sampling of individual pixels renders descriptor sensitive to noise and keypoint location errors, some pre-smoothing is usually applied to improve stability and robustness of the descriptors [34].

In BRIEF [10] the authors investigated six strategies to select intensity test locations, concluding that sampling using isotropic Gaussian $\left(0, \frac{1}{25} S^{2}\right)$ offers minor advantage over uniform, coarse-polar and Census-like sampling. The length of the descriptor is $n_{b}=128,256,512$ bit. The FAST [35] or CenSurE [36] keypoint detectors were used.

ORB [14] builds on the BRIEF design (with FAST detector) using a greedy search algorithm to maximize the variance and to minimize the absolute correlation between selected pairs. Since BRIEF sampling is not designed to be rotationally invariant, the intensity centroid is used as a simple but effective measure of patch orientation. A learning method for decorrelating features under rotational invariance is also applied.

To boost the recognition performance, a discriminative approach can be adopted to learn linear projections that map image patches to a more discriminative subspace. The binary Discriminative BRIEF (D-BRIEF) descriptor [15] is computed by applying a set of orthogonal linear projections and the corresponding thresholds learned from two training sets containing matching $\mathcal{P}$ and non-matching $\mathcal{N}$ image patches.

ALOHA [16] uses a set of patterns, reminiscent of Haar basis functions, to compute intensity differences (Figure 2c). These values are then thresholded to obtain a binary descriptor. A 3-level, hierarchical decomposition of a patch is used and the basis functions are applied at each resolution.

BRISK [11] introduces a new sampling pattern with 59 sampling points located on 4 concentric circles and the central feature point (Figure 2a). Gaussian smoothing with $\sigma$ equal to half the distance between circles is used. Each sampling 


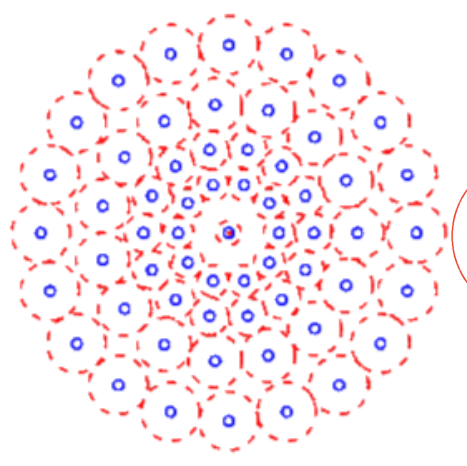

(a)

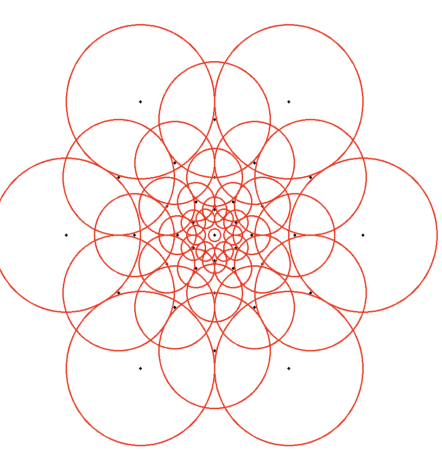

(b)

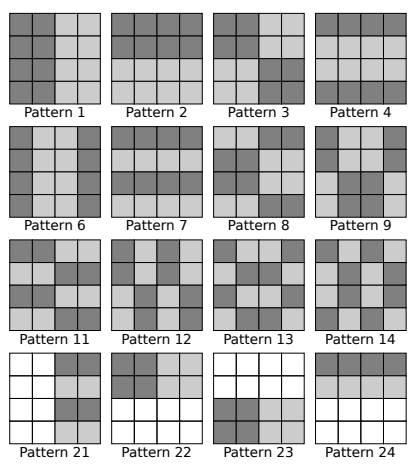

(c)

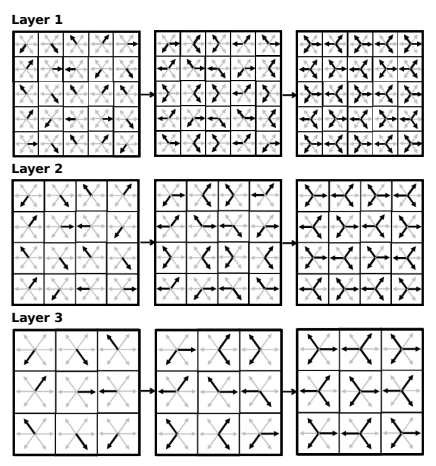

(d)

Fig. 2. Examples of image patch patterns centered on a keypoint for binary descriptors computation. (a) BRISK sampling locations and (b) FREAK sampling locations with red circles representing the standard deviation of the Gaussian kernel used to smooth the intensity values of the sampling points; (c) ALOHA pixel patterns containing two subgroups to perform binary intensity tests; (d) BRIGHT progressive bit selection for hierarchical Histogram of Oriented Gradients (HoG) creation. BRISK, FREAK and BRIGHT pattern pictures are taken from [11], [12] and [13] respectively.

point can participate in several comparisons, reducing memory access during extraction. The patch orientation is estimated as an average long-distance gradient direction.

FREAK [12] sampling pattern is inspired by the human visual system and uses a circular grid with higher density of points near the center. The pairs exhibit a symmetric scheme and the patch orientation is computed in a similar fashion to BRISK (Figure 2b). Authors also vary the size of the Gaussian filter according to the log-polar retinal pattern, and use overlapping receptive fields to improve performance.

LATCH [17] compares the intensity of 3 square sub-patches $\tilde{s}$ in $p$ to produce a single bit of the descriptor. By considering $T$ different triplets $\left\{\tilde{s}_{0}, \tilde{s}_{1}, \tilde{s}_{2}\right\}$, the similarity of $\tilde{s}_{0}$ to the other two sub-patches is given by their Frobenius norm.

2) Gradient-oriented descriptors: This class of binary descriptors uses relations between the distribution of gradient directions and/or magnitudes within the patch, in a similar fashion to SIFT. Histograms of gradient direction are generally more robust, but also more complex to compute.

BRIGHT [13] is extracted by computing a hierarchical Histogram of Oriented Gradients (HoG) in the local patch centered around the keypoint (Figure 2d). At layer 1, the patch is partitioned into square blocks containing a HoG of 6 orientation bins. The bins are aggregated into macroblocks to form a new histogram for layer 2 and then for layer 3. Each histogram element is binarized using an adaptive threshold. High correlation of orientation bins on adjacent blocks is reduced with a progressive bit selection technique, which removes elements corresponding to similar orientations from horizontally and vertically adjacent blocks.

Oriented Gradient Binary (OGB) [18] encodes the local gradient directions and their occurrence locations by mapping the gradient magnitude of every pixel in the current block into corresponding bins of the orientation histogram. Boundary effects are avoided by means of a bilinear interpolation, which distributes the value of each gradient sample into its adjacent histogram bins. Non-linear illumination changes can cause a large change in relative magnitudes: a two-step feature vector normalization is applied so that no entries can be greater than 0.2 . The average value of the resulting feature vector is then used as threshold to quantize each feature into a binary value.

3) Intensity and Gradient hybrids: Some descriptors combine intensity, gradient magnitude and orientation information to increase robustness. FRIF [19] incorporates both local pattern and inter-pattern information by means of pairwise intensities of sampling points around $N$ pattern locations $p_{i}$ with an estimated local dominant orientation $\theta$. Interpattern intensity comparisons are performed between the $M$ shortest rotated pairs $\left(p_{i}^{\theta}, p_{j}^{\theta}\right)$. The size of the descriptor is $n_{b}=M+N=512$ bit.

LDB, for Local Difference Binary [20], directly computes a binary string using difference tests on pairwise spatial bins within the patch. For each bin, a function $\mathcal{F}$, as tuple $\left\{\mathcal{F}_{I}, \mathcal{F}_{d x}, \mathcal{F}_{d y}\right\}$ for local average intensity and gradient response in $x$ and $y$ directions, is computed. Each pair of grids generates 3 bits using binary tests. In case of $N$ grids, the final size is $n_{b}=3 N(N-1) / 2$.

4) Moment-based descriptors: MOBIL [21] uses fundamental moments between patch sub-regions. Five moments are computed for each sub-region: a zero order, two first order and two second order. It uses 56 sub-region comparisons on a $4 \times 4$ grid patch, resulting in a descriptor size $n_{b}=280$.

5) Boosted and Transformed descriptors: Various approaches have been deployed to increase the discriminatory power of descriptors and reduce their size. BAMBrisk [22] considers long- and short-distance intensity tests from the BRISK pattern and applies the Asymmetric Pairwise Boosting algorithm [37] to obtain a binary output with size up to $n_{b}=512$. A classifier is learned with a training based on sets of matching and non-matching pairs.

BinBoost [23] is also built using labeled image patches, in order to reduce the Hamming distances between descriptors of patches from positive pairs. The classifiers are optimized iteratively, maximizing the weighted correlation of their output and the data labels. Weak learners are parametrized by a rectangular region over the patch, an orientation for the intensity gradient, and a threshold.

MPEG CDVS-TM standard [38] applies a linear transform coding to SIFT descriptors. It uses sums and differences of adjacent gradient bins in the SIFT descriptor in a alter- 
nating fashion, combined with the ternary quantization of the transformed coefficients. A customized distance function substitutes classic Hamming distance function.

Simonyan et al. [39] uses convex optimization to learn good pooling regions for descriptor extraction and to select optimal dimensionality reduction. The learned low-dimensional realvalued descriptors are converted to binary by means of a linear projection to a higher-dimensional space (Parseval tight frame expansion) followed by thresholding. We will refer to this descriptor as SVZ, after the authors' initials.

6) CNNs methods: Despite the popularity of Convolutional Neural Networks for image retrieval, very few works on binary local descriptors have been published. DeepBit [40] learns compact binary descriptors in an unsupervised manner. The parameters of the network are updated using backpropagation with three criteria: (i) to minimize the loss quantization, (ii) to reduce the bit correlation and (iii) to evenly distribute the binary codes. In Deeply Learned Feature Transform (DELFT) [41], Euclidean and Hamming embedding for image patch description is trained with triplet convolutional networks. These features can cooperate with existing nearest neighbor search algorithms in Euclidean and Hamming space, improving the speed-accuracy tradeoff.

The current CNN-based designs are too computationally complex for mobile applications (approximately three orders of magnitude compared to BRISK). Furthermore, since their accuracy is comparable to the SIFT and SVZ benchmarks presented here, the performance gain over the fast binary descriptors is limited.

\section{EXISTING EVALUATION PROTOCOLS AND DATASETS}

Before we introduce our descriptor characterization framework, we critically review the existing evaluation protocols and associated datasets. Evaluation protocols can be classified into three groups.

- Patch-level evaluation uses a collection of independent image patches (e.g. Liberty and Yosemite [42]) ready for descriptor extraction. Therefore, they do not require any keypoint detection stage.

- Database-level evaluation specifies query images and a database (e.g. Holidays [43] and Oxford [44]) for retrieval of relevant images.

- Image-level evaluation is based on a small set of transformed or distorted images with respect to a reference image (e.g. Graffiti [45]).

Patch-level evaluation is usually simple because it only requires the analysis of the distances between the matching and non-matching descriptor pairs, e.g. in a form of an ROC curve. Nevertheless, matching results may be biased depending on the keypoint detection stage used to produce the patches. The latter two categories require more sophisticated measures to determine the level of similarity between two images, such as the nearest neighbor distance ratio test or simply ratio test [46]. Missing detection of a single matching pair of keypoints does not have an immediate impact in the overall performance, since many matching pairs are usually returned.

During our study, it became clear that existing protocols do not reflect the challenges typical for mobile applications. The
TABLE I

WINNING ( $\bullet$ ) AND AVAILABLE DESCRIPTORS (O) FOR DIFFERENT PAPERS BF: BRIEF, OB: ORB, BK: BRISK, FK: FREAK, SF: SURF, CE: CENSURE.

\begin{tabular}{|c||c|l||l||c|c|c|c|}
\hline Cit. & Type & Dataset & Detect. & BF & OB & BK & FK \\
\hline \hline$[47]$ & Patch & Notredame [42] & - & $\circ$ & $\bullet$ & $\circ$ & 0 \\
{$[47]$} & DB & Oxford [44] & SF, BK & $\circ$ & $\circ$ & $\bullet$ & $\circ$ \\
{$[47]$} & DB & ZuBuD [48] & SF, BK & $\circ$ & $\circ$ & $\bullet$ & $\circ$ \\
{$[47]$} & DB & CTurin180 [49] & SF, BK & $\circ$ & $\circ$ & $\bullet$ & $\circ$ \\
{$[50]$} & Image & Graffiti [45] & SF & $\circ$ & $\circ$ & $\bullet$ & \\
{$[51]$} & Image & Graffiti [45] & Several & $\bullet$ & $\circ$ & $\circ$ & \\
{$[52]$} & Image & Graffiti [45] & OB & $\circ$ & $\circ$ & $\circ$ & $\bullet$ \\
{$[53]$} & Image & Bark [45] & SF & $\circ$ & $\circ$ & $\bullet$ & $\circ$ \\
{$[53]$} & DB & Stanford [54] & SF & $\circ$ & $\circ$ & $\bullet$ & $\circ$ \\
{$[55]$} & DB & Stanford [54] & CE, OB & & $\circ$ & $\bullet$ & $\circ$ \\
\hline
\end{tabular}

datasets used (e.g. [43], [44]) contain pictures of good quality. In contrast, mobile scenarios often involve significant motion of the device or objects in the scene, resulting in motion blur. Furthermore, objects of interest are frequently small and exhibit various distortions, such as shadows, transparency, nonrigid deformations or dramatic reflections and illumination changes. We therefore acquired a new dataset for testing the behavior of descriptors in more challenging scenarios, which are frequent in mobile use (see Sections IV-C and V-D).

Different matching strategies and metrics have been used in previous reviews. Precision and recall are the most common for patch-level and image-level matching performance [47], [50], [51], [53], while mean average precision (mAP) is frequently used for retrieval performance at database-level [47], [50]. In order to make search more challenging, distractor features may be introduced [50]. In [55] accuracy is defined as percentage of query images correctly classified.

When a protocol requires a database retrieval, the specific image ranking algorithm does have a major impact on the performance. In [50] fast matching is done using multiple randomized kd-trees with $\epsilon$-approximate nearest neighbor ( $\epsilon$ ANN) search [56]. In [51] matching score is based on the ratio between the number of matching descriptors, confirmed by geometric verification, and the total number of features. In [55] multi-probe Locally Sensitive Hashing [57] for the nearest neighbor search is used. In [52] homography information is provided and the percentage of valid matches out of the total number of expected matches is computed. In [53] RANSAC [58] is used to confirm matching keypoints pairs, while the average number of best matches per descriptor is defined as product between the average number of keypoints and the ratio between the number of best matches and number of keypoints which passed the ratio test. Geometric verification is often too complex for mobile use, therefore we employed a simpler procedure based on the numbers of matching keypoints (see Section IV-B).

Results of the prior evaluations are summarized in Table I. Most of the reviews are limited to a narrow subset, typically including BRIEF, ORB, BRISK and FREAK binary descriptors. While we note that most papers consider BRISK as the best performing descriptor, this view is not unanimous. According to [55], BRISK, ORB and FREAK show very similar accuracy and can compete well with SIFT, in terms of distinctiveness. In [50] BRIEF outperforms ORB, and BRISK is comparable to 
SIFT, while in [52] BRISK and FREAK outperform both ORB and BRIEF. [47] and [53] confirm BRISK on top of ORB, BRIEF and FREAK. Following the detector/descriptor pairings evaluation, [51] highlights that best overall performance does not always correspond to the original authors' recommendations. For perspective transforms, BRIEF leads in recall and matching score outperforming ORB and BRISK, despite the descriptor simplicity, while BRISK still leads in precision metric. It is difficult to reconcile all these results because, as shown in the table, different datasets and methodologies were used. Furthermore, another level of complexity is added by the impact of keypoint detectors on the descriptor performance. Detectors listed in the table are the ones used by the winning descriptors. While BRISK and ORB have their own embedded detector, other techniques use other well-known detectors such as FAST, CenSurE, SIFT and SURF [25].

Some prior evaluations measure matching accuracy at an arbitrarily selected False Positive Rate, which could lead to a bias. For instance, [11], [12], [46], [47] use FPR $=0.1$, however for $10^{4}$ descriptors typically present in every image, such FPR would produce $10^{3}$ false matches! High number of false matches requires costly verification procedure, such as RANSAC, and may contribute to poor performance. In our evaluation, we use much lower FPR values (e.g. $5 \cdot 10^{-5}$ ), leading to different and sometimes unexpected conclusions on relative merits of various descriptors and optimal working points. As descriptors' performance depends on the matching approach, in the next section we evaluate several lightweight strategies, based either on thresholding of the descriptor distances or the distance ratios, combined with either onedirectional or bi-directional matching.

\section{EVALUATION FRAMEWORK}

In this section we outline our comprehensive evaluation framework, which includes selected existing protocols, datasets and operating conditions, but also adds new tests to address the identified deficiencies. We used two different detectors, one simple and fast (BRISK) and one more robust but slow (Hessian-Affine), to determine the upper-bound of the performance. We also fill the gaps in the list of all the previous mentioned binary descriptors, and introduce a new video sequence dataset to challenge the robustness of the descriptors on different real artifacts, deformations, motion and illumination issues.

\section{A. Descriptors}

For our evaluations, we selected 21 state-of-the-art binary descriptors. New methods are constantly published, therefore we determined some criteria for the selection of the algorithms to include in the framework. We did not consider: (1) methods built on the computation of auxiliary spatial features between correlated keypoints, since the proposed descriptor extraction stage takes independent patches as input for each keypoint; (2) methods depending on the geometric relations between the descriptors to validate the matches, since the matching stage is based on fast implementations. Furthermore, we considered the algorithms with source code available online, under request, or correctly implementable using the published information only.

We also included 6 top-performing non-binary descriptors, as indicator of the upper-bound performance limits: SIFT, SURF, LQ-HoG [26], LIOP [27], MRRID, and MROGH [59].

ALOHA, BRIGHT, LDB, LQ-HoG and OGB descriptors have been implemented according to the corresponding papers. The OpenCV library [60] was used for SIFT, SURF, BRIEF, ORB, FREAK and BRISK descriptors, while LIOP is from the VLFeat library [61]. For all other methods, their authors kindly provided the source code. For LQ-HoG the 135-bit configuration was used as shown in [26], while for OGB the $4 \times 4$ grid cells pattern with 16 gradient orientations and no down-sampling was selected [18].

We also introduced a modified version of BRIGHT descriptor, called $\mathrm{BRIGHT}^{+}$, which adds a forth layer for the hierarchical HoG by means of a dedicate threshold $\alpha=0.13$. This leads to a marked improvement in performance with only a marginal size increase to 162 bits.

\section{B. Pipeline and matching strategies}

Figure 3 shows a simple pipeline for database-level evaluation. Different similarity metrics between image $I_{a}$ and $I_{b}$ are computed using the following steps.

1) Keypoint detection: The first stage involves image keypoint detection using a set of $v$ keypoint detectors $K_{1}, K_{2}, \ldots K_{v}, \in K . N_{a}$ and $N_{b}$ are the number of keypoints in $I_{a}$ and $I_{b}$ for a generic detector $K_{h}$, where $1 \leq h \leq v$.

2) Patch creation: Based on the scale and location of detected keypoints, a set of normalized patches is created for each image. Rotational ambiguity is eliminated by rotating the patch according to the direction indicated by the detector. For $I_{a}$, this results in a canonical form $p\left(I_{a}, K_{h}, k_{i}\right)$, where $i$ is the index of a keypoint, $1 \leq i \leq N_{a}$.

3) Descriptor extraction: Given a set of $w$ descriptor algorithms $\delta_{1}, \delta_{2}, \ldots, \delta_{w} \in \Delta$, patch $p\left(I_{a}, K_{h}, k_{i}\right)$ and algorithm $j(1 \leq j \leq w)$ are the input of the extraction routine for the computation of a descriptor $d\left(I_{a}, K_{h}, k_{i}, \delta_{j}\right)$. For simplicity, after setting an arbitrary detector $h$ and descriptor algorithm $j$, let $d\left(I_{a}\right)$ and $d\left(I_{b}\right)$ be the list of descriptors in $I_{a}$ and $I_{b}$.

4) Distance computation: $d\left(I_{a}\right)$ and $d\left(I_{b}\right)$ are used to build a Hamming or Euclidean distance table with size $N_{a} \times N_{b}$. Each element in the table represents the descriptor distance between a keypoint in $I_{a}$ and a keypoint in $I_{b}$.

5) Similarity computation: The typical method to detect matching descriptors from $d\left(I_{a}\right)$ and $d\left(I_{b}\right)$ is the ratio test. For each descriptor in $d\left(I_{a}\right)$, the ratio between its distance $\gamma_{a b}^{\prime}$ to the closest descriptor $d_{a \rightarrow b}$ in $d\left(I_{b}\right)$ and its distance $\gamma_{a b}^{\prime \prime}$ to the second closest one is computed. If the ratio is less than a threshold $\tau$, the test is passed. Geometric consistency check is usually applied on the locations of the corresponding keypoints. This operation is not possible in many mobile applications because the verification is computationally demanding. Therefore, we focus on low-complexity matching strategies.

Let $R_{a}=\frac{\gamma_{a b}^{\prime}}{\gamma_{a b}^{\prime \prime}} \leq 1$ be the ratio distance to test. ${ }^{1}$ Considering

${ }^{1}$ For time efficiency, in case of non-binary descriptors the ratio between the squared L2 distances is considered. This choice affects the values of parameter $\tau$ in Table III. 


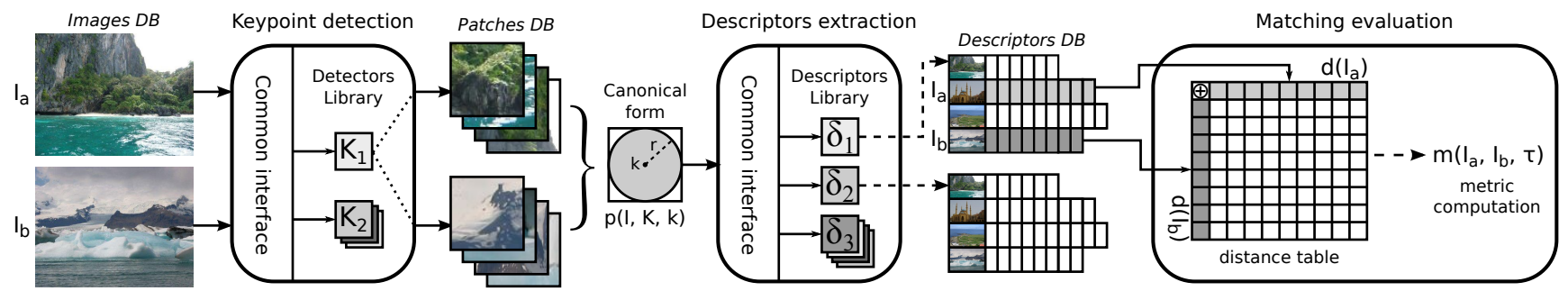

Fig. 3. Pipeline for similarity evaluation between images. A patch database $p(I, K, k)$ is created for each image $I$ and for each keypoint $k$ extracted by a detector $K$. Each $p(I, K, k)$, characterized by a fixed radius $r$ and scale and rotation invariance according to the keypoint information, is used to compute a single descriptor $d(I, K, k, \delta)$ where $\delta$ is a descriptor class from a descriptors library $\Delta=\left\{\delta_{1}, \delta_{2}, \delta_{3}, \ldots\right\}$. Given an image pair $\left\{I_{a}, I_{b}\right\}$, for each $K, k$ and $\delta$, the matching evaluation stage consists in the computation of the distance table of all their descriptors $d\left(I_{a}\right)$ and $d\left(I_{b}\right)$ and the extraction of a matching metric $m$ according to a threshold $\tau$ for the nearest neighbor ratio test.

the closest matching descriptors $d_{a \leftarrow b}$ to $d\left(I_{b}\right)$ in $d\left(I_{a}\right)$ we can also define $R_{b}=\frac{\gamma_{b a}^{\prime}}{\gamma_{b a}^{\prime \prime}} \leq 1$. Six different cumulative functions $\xi$ are proposed depending on the direction of the ratio test (i.e. $\vec{\xi}$ and $\overleftrightarrow{\xi}$ ), the weight assignment for $R$ and $\tau$ values (i.e. $\xi_{1}$ and $\left.\xi_{\alpha}\right)$ or the use of Hamming distance $\left(\xi_{h}\right)$. Function $\vec{\xi}$ adds a candidate descriptors pair $d_{a b} \doteq\left\{d\left(I_{a}\right), d\left(I_{b}\right)\right\}$ to the matching list if $R_{a} \leq \tau$, while $\overleftrightarrow{\xi}$ checks if:

$$
R_{a} \leq \tau \wedge R_{b} \leq \tau \wedge d_{a \rightarrow b}=d_{a \leftarrow b} .
$$

While $\xi_{1}$ simply gives a weight 1 to all the descriptors pairs which passed the ratio test, $\xi_{\alpha}$ gives a weight $\alpha$ as follows:

$$
\alpha= \begin{cases}1 & \text { if } R \leq \frac{\tau}{2} \\ \frac{1}{2}+\frac{\tau-R}{\tau} & \text { if } \frac{\tau}{2}<R \leq \tau \\ 0 & \text { if } R>\tau .\end{cases}
$$

$R=R_{a}$ in case of $\xi=\vec{\xi}$, otherwise $R=\max \left(R_{a}, R_{b}\right)$. The weight $\alpha$ gives more importance to descriptor pairs more distant to other candidates, enabling the selection of the most robust matching pairs. Therefore, the final matchability score $\alpha\left(d_{a b}\right)$ for a group of matching descriptor pairs $\left\{d_{a b}\right\}$ in case of $\xi_{\alpha}$ test is:

$$
\alpha\left(d_{a b}\right)=\sum_{i}^{\left|d_{a b}\right|} \alpha_{i} .
$$

In case of evaluation of binary descriptors with size $n_{b}$, a $\xi_{h}$ function is also introduced for absolute Hamming distance test. Given a Hamming threshold $h^{*}$, with $0 \leq h^{*} \leq 1, \overrightarrow{\xi_{h}}$ test gives a weight 1 to all the descriptors pairs with distance $H$ satisfying $H \leq h^{*} \cdot n_{b}$, while $\overleftrightarrow{\xi_{h}}$ test also checks if $d_{a \rightarrow b}=$ $d_{a \leftarrow b}$.

6) Relevant images ranking: Relevant images retrieved from queries in the datasets [43], [44] need to be ranked to compute certain overall performance index, such as mAP. We define the following image similarity metric $m$ :

$$
m\left(I_{a}, I_{b}, \tau\right)=\frac{\xi\left(I_{a}, I_{b}, \tau\right)}{\mu\left(N_{a}, N_{b}\right)}
$$

where $\mu\left(N_{a}, N_{b}\right)$ is a normalization function depending on the number of descriptors in $I_{a}$ and $I_{b}$. Three different $\mu$ functions are tested: $\mu_{\text {min }} \doteq \min \left(N_{a}, N_{b}\right), \mu_{\sqrt{ }} \doteq \sqrt{N_{a} N_{b}}$ as suggested in [29], and $\mu_{1} \doteq 1$ for no normalization.

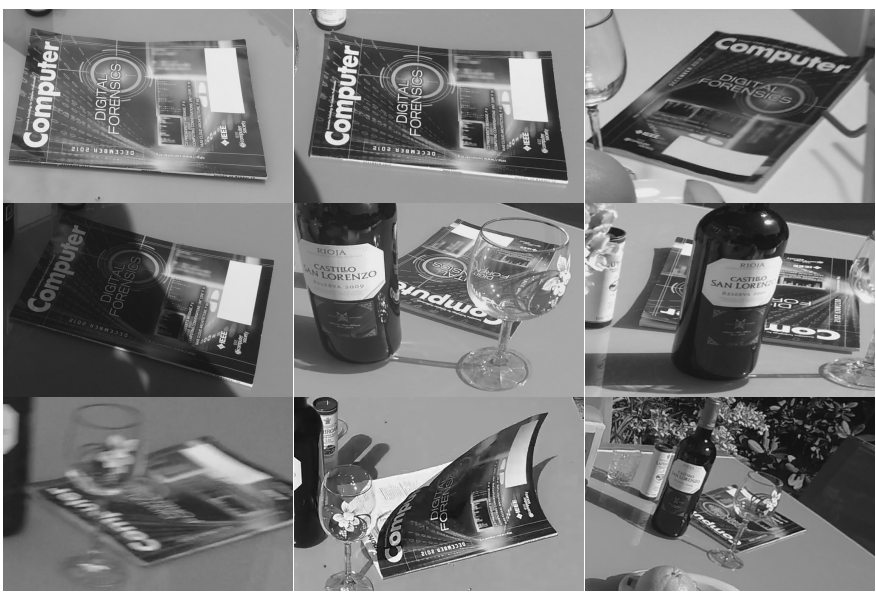

Fig. 4. Regions of interest extracted from the original frames for visualization purposes only, showing different transformations for video frames containing the query Magazine. Top row: three different illumination changes and reflections (on the right with artificial illumination). Middle row: shadowing, transparency and occlusion. Bottom row: blurring, deformation and zoom out.

\section{Mobile dataset}

We propose a new video dataset for objects retrieval and recognition called Surrey Mobile [62]. It contains scenes depicting common objects such as postcards, boxes, papers, flowers, glasses, bottles, business cards and magazines. Our dataset is designed to mimic the challenges of object recognition in mobile scenario: 1) it uses different mobile devices for data acquisition, 2) the video recording introduces compression artifacts and 3) the ad-hoc video setup requires robust matching capabilities to face the image distortions.

Some examples of the demanding complexity are shown in Figure 4. Videos were recorded at different times of the day, with natural and artificial light generating different shadows and reflections on materials such as paper, plastic and glass. Videos were also subject to significant degree of motion blur and zoom of the target objects: these factors test to the extreme the robustness of keypoints detection and the descriptors capability. The objects were also arranged to create occlusion and transparency effects. Moreover, the object queries were chosen to promote false positive matches of local image features such as alphabetical letters and geometric forms. See Section V-D for full details on performance. 


\section{EXPERIMENTAL RESULTS}

In this section we evaluate the 21 binary descriptors using patch-level, database-level and image-level evaluation protocols. We start with the patch-level evaluation as it gives an initial idea about the strength of descriptors and illustrates the behavior of matching and non-matching distance distributions. In database-level evaluation we determine the optimum ratio thresholds $\tau$ and distance thresholds $h^{*}$, which are then applied in image-level evaluation and to the proposed mobile dataset. Results are grouped into three categories according to the descriptor size: compact $[128 \div 162]$, medium $[256 \div 280]$ and large $[486 \div 512]$. We also present results for the ultra-compact D-BRIEF descriptor (32 bit) to illustrate the impact of the descriptor size on matching performance. For each category the best descriptors are highlighted. The results for CDVS-TM (based on SIFT) and SVZ (based on real-valued descriptors) are kept as reference due to their higher descriptor extraction complexity, which may result in superior matching performance. SVZ provided the descriptor modeling and extraction code for several values of $n_{b}$ and training datasets. For patchlevel experiments $n_{b}=\{128,512,1024\}$ were evaluated, while for the image-level experiments $n_{b}=512$ on training dataset Notredame was used as it gives the best results.

\section{A. Patch-level matching performance}

In this section we evaluate descriptors based on scalenormalized $64 \times 64$ pixels patches from the MPEG Compact Descriptors for Visual Search (CDVS) dataset [26] and the Winder and Brown dataset [42]. These datasets contain 100K and $500 \mathrm{~K}$ matching pairs respectively and consist of canonical scaled and oriented patches around Difference-of-Gaussian interest points. To incorporate interest point jitter statistics, patches were warped using small random similarity warps affecting position, rotation and scale. The standard deviation of synthetic noise added to the CDVS dataset is 0.4 pixel, 11 degrees and $2^{0.12}$ octaves in orientation, shift and scale respectively. The Winder and Brown dataset considers a pair as matching if the detected keypoint is within 5 pixels position, 22.5 degrees rotated and 0.25 octaves of scale. For each dataset, the number of non-matching pairs considered is 100 times greater than the number of matching pairs.

Default patch smoothing is used for OpenCV descriptors, while for the other descriptors a Gaussian smoothing of $\sigma=2.7$ pixels is applied to the patch, as suggested in [1]. Table II summarizes the matching results. Three different metrics are considered: KL divergence for matching and nonmatching Hamming distance distributions and true positive rate (TPR) values at two FPR operating points $\left(10^{-2}\right.$ and $5 \cdot 10^{-5}$ ). KL divergence represents an averaging view across different FPR points. We note that this metric is better in predicting performance at $\mathrm{FPR}=0.01$ than at lower operating points. For instance, KL divergence fails for FREAK, which has the highest value on CDVS dataset, while TPR values are about average. Conversely, BAMBrisk ${ }_{256}$ has a low KL divergence but high TPR at low FPR. Therefore, to understand true behavior at very low FPR, ROC curves should be used, while KL divergence can be considered for fast matching performance evaluation. In the $[128,162]$ size range, BRIGHT $^{+}$ is the highest ranked descriptor, while in the 256 bit category BinBoost presents the highest KL divergence and TPR values for all the datasets. However, BRIGHT + is not wide behind on CDVS at FPR $=0.01$. In $[486,512]$ size range BRISK shows the best performance for all the dataset at FPR $=0.01$ and for Notredame and Yosemite at FPR $=5 \cdot 10^{-5}$. Furthermore, SVZ performance appears to saturate at 512 bit, as the 1024 bit version does not improve TPR values. Regarding non-binary descriptors, for all the dataset at FPR $=10^{-2}$ SIFT has the best performance ( $94 \%$ on CDVS), while at FPR $=5 \cdot 10^{-5}$ LIOP works better ( $64 \%$ on CDVS). ROC curves are plotted in the extended FPR range $\left[10^{-4}, 1\right]$ and shown in Figure 5.

\section{B. Database-level matching performance}

To assess the matching performance of the descriptors on real images, two different datasets are used. The Oxford Buildings dataset [44] consists of 5062 images collected from Flickr by searching for particular Oxford landmarks. A bounding box containing the relevant object was manually annotated for 55 queries. The Holidays dataset [43] contains images from different scene types to test the robustness to transformations such as rotation, viewpoint and illumination changes, blurring. This dataset consists of 500 queries representing distinct scenes or objects and 991 corresponding relevant images.

Features were extracted by means of Hessian-Affine [63] and BRISK detectors to create $64 \times 64$ pixels normalized patches. The Hessian-Affine detector is acknowledged as robust to complex image transformations [64], while BRISK keypoints are very fast to extract. A series of brute-force matching experiments were conducted in order to find the best thresholds for ratio test $\tau$ and absolute Hamming distance $h^{*}$ from a range $[0.05,0.90]$ with step 0.01 in different operating conditions. Table III shows mAP results for $\xi_{\alpha}$ and $\xi_{h}$ test functions on both Holidays and Oxford datasets for keypoints extracted using the Hessian-Affine detector. The ranking of the descriptors is slightly changed from the previous patch-level evaluation and D-BRIEF results are not reported because of the poor performance. Among the three type of normalization functions $\mu$, in case of no normalization $\left(\mu_{1}\right)$ the best results were obtained. For instance, by choosing the function test $\overleftrightarrow{\xi_{\alpha}}$, which gives the highest mAP values, $\mu_{1}$ test shows between $2 \%$ and $3 \%$ better results than $\mu_{\sqrt{ }}$ and between $12 \%$ and $23 \%$ than $\mu_{\text {min }}$. Because of the considerable amount of data, results from these two normalization functions are omitted. $\xi_{\alpha}$ test outperforms $\xi_{1}$ test for both bidirectional and unidirectional test by a value between $0.5 \%$ and $2 \%$. mAP values between $\overrightarrow{\xi_{h}}$ and $\overleftrightarrow{\xi_{h}}$ test are very similar, therefore the bidirectional test could be avoided, while keeping the same optimum $h^{*}$ value. A valuable result concerns the directionality of $\xi_{\alpha}$ test. $\overleftrightarrow{\xi_{\alpha}}$ outperforms $\overrightarrow{\xi_{\alpha}}$ between $5 \%$ and $25 \%$. $\overleftrightarrow{\xi_{\alpha}}$ is a more demanding test, since it has to be satisfied in both the directions, therefore the ratio threshold $\tau$ tends to increase, i.e. the difference between $\left\|d_{a}, d_{b}^{\prime \prime}\right\|$ and $\left\|d_{a}, d_{b}^{\prime}\right\|$ tends to increase. For instance, for BRISK, FRIF and BAMBrisk ${ }_{512}$ on 


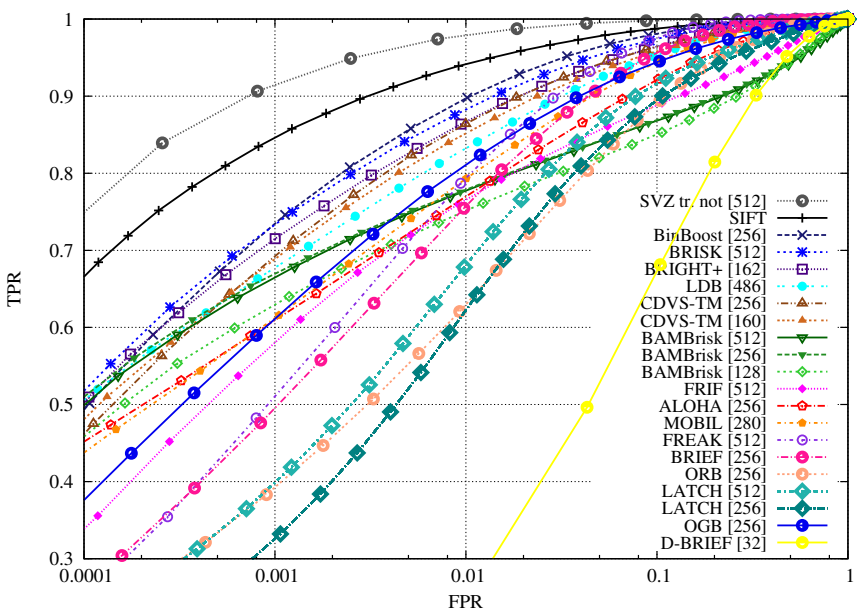

(a) CDVS dataset

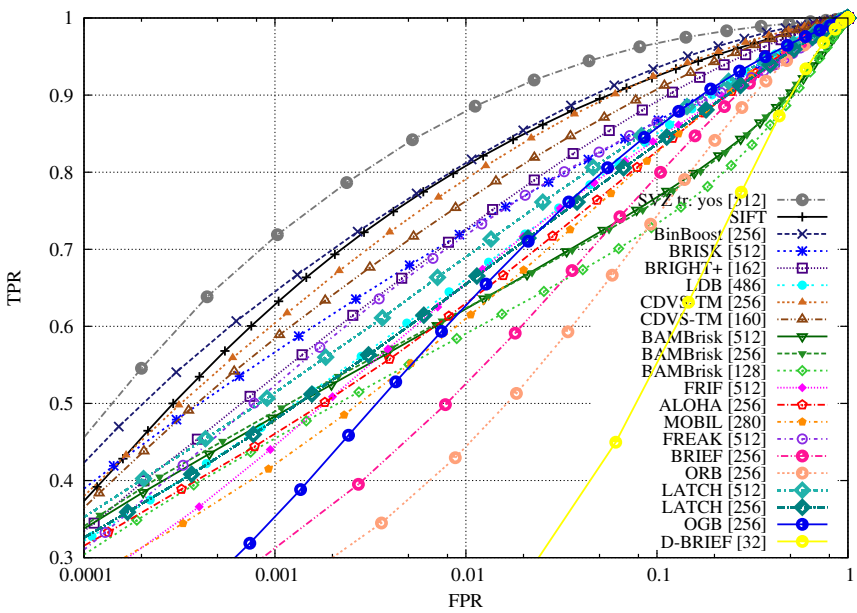

(c) Notredame dataset

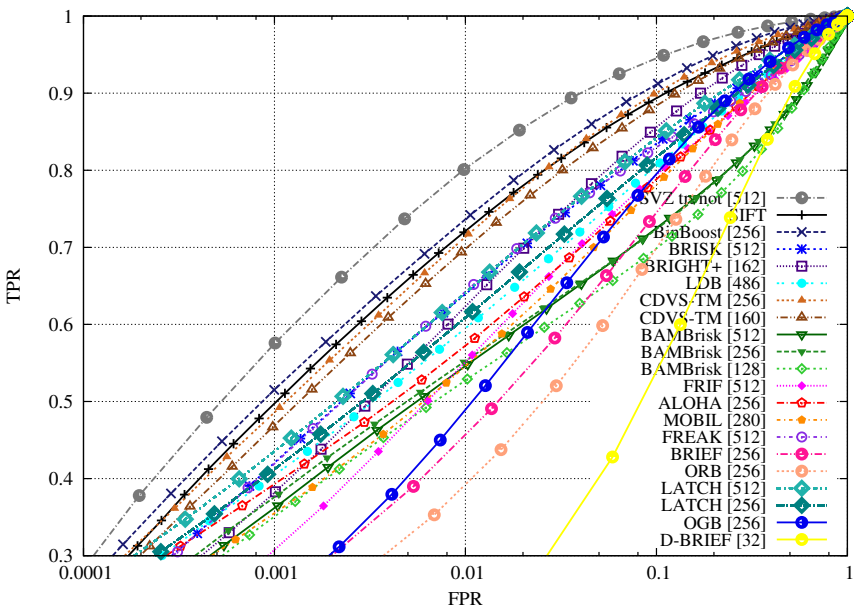

(b) Liberty dataset

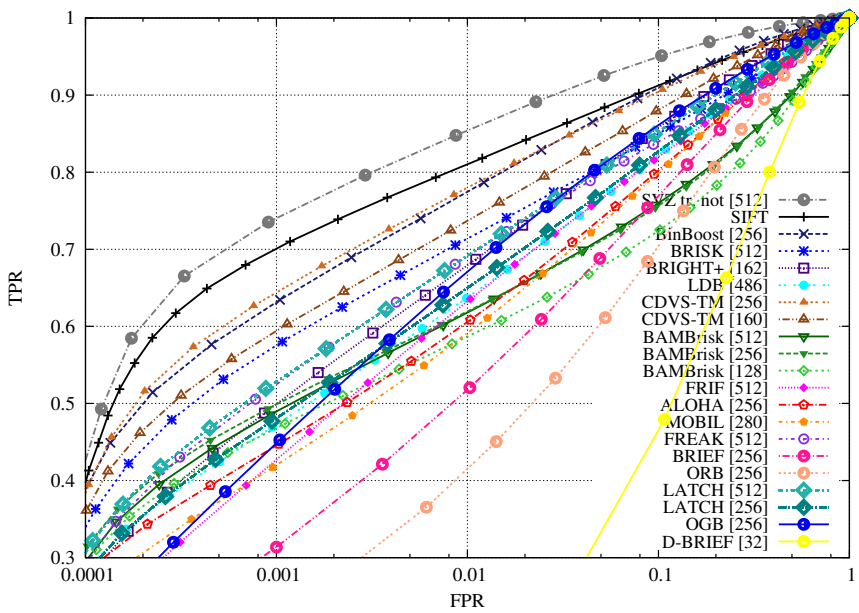

(d) Yosemite dataset

Fig. 5. Comparison of ROC curves at patch level for different binary descriptors on CDVS, Liberty, Notredame and Yosemite datasets. Descriptor size in squared parentheses. SIFT is added as reference.

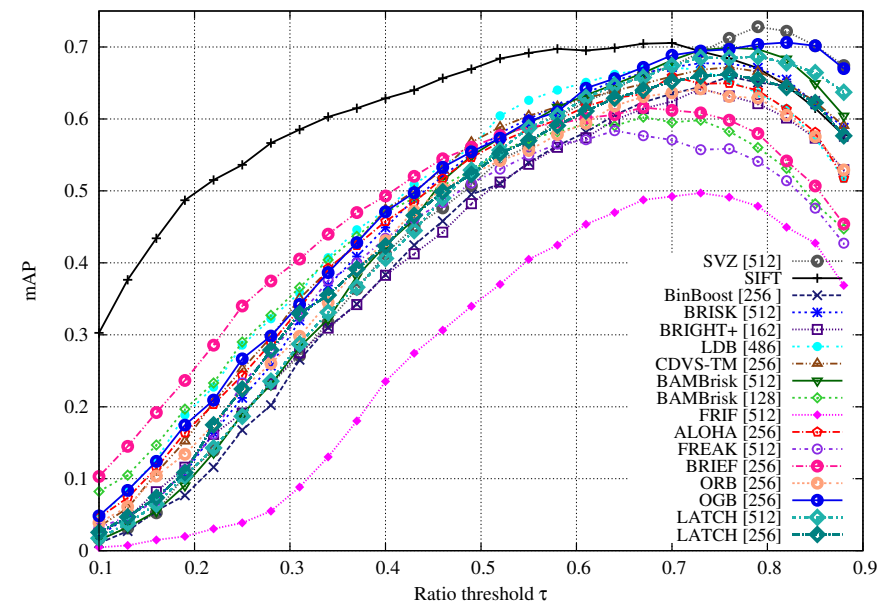

(a)

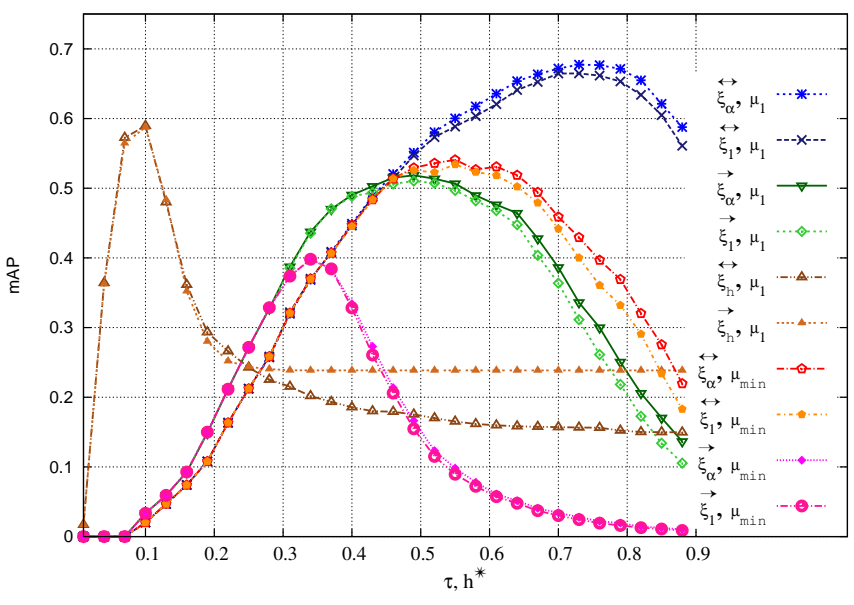

(b)

Fig. 6. (a) mAP values by varying ratio threshold $\tau$ for $\overleftrightarrow{\xi_{\alpha}}$ matching test with $\mu=1$ on Holidays dataset. (b) mAP results (BRISK descriptor) by varying both ratio threshold $\tau$ and Hamming absolute threshold $h^{*}$ for several combinations of matching tests $\xi$ and normalization factors $\mu$ on Holidays dataset. 
TABLE II

KL DIVERGENCE FOR MATCHING / NON-MATCHING HAMMING DISTANCE DISTRIBUTIONS AND TPR VALUES FOR CDVS, NOTREDAME, LIBERTY AND YOSEMITE DATASETS FOR BINARY AND NON-BINARY $(*)$ DESCRIPTORS.

\begin{tabular}{|c|c|c|c|c|c|c|c|c|c|c|c|c|c|}
\hline \multirow{2}{*}{ Descriptor } & \multirow{2}{*}{ Size } & \multicolumn{4}{|c|}{ KL divergence } & \multicolumn{4}{|c|}{ TPR @FPR = 0.01} & \multicolumn{4}{|c|}{ TPR @ $@$ FPR = 0.00005} \\
\hline & & CDVS & LIB & NOT & YOS & CDVS & LIB & NOT & YOS & CDVS & LIB & NOT & YOS \\
\hline D-BRIEF [15] & 32 & 2.785 & $\overline{1.640}$ & 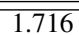 & 1.255 & 0.24 & $\overline{0.15}$ & $\overline{0.16}$ & $\overline{0.11}$ & 0.00 & $\overline{0.00}$ & 0.00 & $\overline{0.00}$ \\
\hline BAMBrisk [22] & 128 & 6.899 & 3.696 & 4.739 & 4.623 & 0.75 & 0.53 & 0.59 & 0.59 & 0.39 & 0.12 & 0.25 & 0.15 \\
\hline SVZ [39] & 128 & 14.969 & 7.411 & - & 8.391 & 0.95 & 0.77 & - & 0.82 & 0.51 & 0.17 & - & 0.18 \\
\hline BRIGHT [13] & 150 & 10.058 & 5.324 & 6.599 & 5.858 & 0.86 & 0.61 & 0.71 & 0.66 & 0.42 & 0.13 & 0.26 & 0.14 \\
\hline CDVS-TM [38] & 160 & 9.500 & 6.161 & 7.347 & 6.898 & 0.85 & 0.70 & 0.76 & 0.74 & 0.40 & 0.15 & 0.29 & 0.17 \\
\hline BRIGHT $^{+}$ & 162 & 10.294 & 5.420 & 6.791 & 6.031 & 0.87 & 0.62 & 0.73 & 0.68 & 0.42 & 0.13 & 0.27 & 0.14 \\
\hline BRIEF [10] & 256 & 9.769 & 3.702 & 4.533 & 4.212 & 0.76 & 0.46 & 0.53 & 0.52 & 0.20 & 0.08 & 0.13 & 0.09 \\
\hline ORB $[1$ & 256 & 6.824 & 3.203 & 3.883 & 3.387 & 0.63 & 0.39 & 0.44 & 0.41 & 0.18 & 0.07 & 0.11 & 0.07 \\
\hline ALOHA [16] & 256 & 8.395 & 4.754 & 5.784 & 5.217 & 0.77 & 0.57 & 0.63 & 0.61 & 0.40 & 0.17 & 0.27 & 0.16 \\
\hline CDVS-TM [38] & 256 & 9.633 & 6.532 & 7.769 & 7.545 & 0.86 & 0.71 & 0.79 & 0.78 & 0.38 & 0.17 & 0.30 & 0.18 \\
\hline BAM & 256 & 7.406 & 4.015 & 5.211 & 5.014 & 0.78 & 0.55 & 0.62 & 0.62 & 0.43 & 0.14 & 0.29 & 0.16 \\
\hline BinB & 256 & 11.767 & 6.912 & 8.248 & 7.602 & 0.90 & 0.74 & 0.81 & 0.77 & 0.40 & 0.18 & 0.34 & 0.18 \\
\hline LATCH [17] & 256 & 5.740 & 5.131 & 5.966 & 5.552 & 0.62 & 0.61 & 0.65 & 0.65 & 0.12 & 0.18 & 0.28 & 0.16 \\
\hline OGB [18] & 256 & 8.321 & 4.120 & 5.167 & 5.669 & 0.81 & 0.49 & 0.63 & 0.67 & 0.30 & 0.06 & 0.10 & 0.12 \\
\hline MOBIL & 280 & 9.480 & 4.475 & 5.508 & 5.029 & 0.79 & 0.55 & 0.61 & 0.59 & 0.38 & 0.14 & 0.23 & 0.15 \\
\hline LD & 486 & 10.139 & 4.922 & 6.114 & 5.503 & 0.83 & 0.59 & 0.66 & 0.64 & 0.44 & 0.16 & 0.28 & 0.15 \\
\hline FREAK [12] & 512 & 12.709 & 5.102 & 6.202 & 5.835 & 0.79 & 0.64 & 0.72 & 0.69 & 0.18 & 0.14 & 0.25 & 0.15 \\
\hline BRISK [11] & 512 & 11.756 & 5.192 & 6.675 & 6.298 & 0.88 & 0.64 & 0.72 & 0.71 & 0.43 & 0.14 & 0.33 & 0.17 \\
\hline FRIF [19] & 512 & 6.935 & 4.237 & 5.603 & 5.088 & 0.77 & 0.55 & 0.66 & 0.63 & 0.27 & 0.09 & 0.22 & 0.12 \\
\hline BAMBrisk [22] & 512 & 7.327 & 3.937 & 5.226 & 4.950 & 0.78 & 0.55 & 0.62 & 0.62 & 0.43 & 0.13 & 0.29 & 0.16 \\
\hline & 512 & 6.578 & 5.507 & 6.467 & 6.090 & 0.68 & 0.64 & 0.69 & 0.69 & 0.17 & 0.19 & 0.31 & 0.16 \\
\hline SVZ $[3$ & 512 & 17.623 & 8.023 & - & 9.184 & 0.98 & 0.80 & - & 0.85 & 0.65 & 0.19 & - & 0.18 \\
\hline SVZ [39] & 1024 & 18.142 & 8.148 & - & 9.448 & 0.98 & 0.81 & - & 0.85 & 0.65 & 0.19 & - & 0.19 \\
\hline SURF [25] & $64^{*}$ & - & - & - & - & 0.89 & 0.61 & 0.68 & 0.67 & 0.41 & 0.10 & 0.21 & 0.16 \\
\hline SIFT [33] & & - & - & - & - & 0.94 & 0.72 & 0.81 & 0.81 & 0.57 & 0.15 & 0.29 & 0.17 \\
\hline LQ-HoG [26] & & - & - & - & - & 0.87 & 0.61 & 0.72 & 0.69 & 0.46 & 0.12 & 0.24 & 0.14 \\
\hline LIOP [ & & - & - & - & - & 0.94 & 0.66 & 0.75 & 0.70 & 0.64 & 0.21 & 0.33 & 0.19 \\
\hline MROGH [59] & $192^{*}$ & - & - & - & - & 0.92 & 0.59 & 0.69 & 0.65 & 0.61 & 0.18 & 0.30 & 0.18 \\
\hline MRRID [59] & $256^{*}$ & - & - & - & - & 0.89 & 0.61 & 0.69 & 0.63 & 0.56 & 0.20 & 0.27 & 0.17 \\
\hline
\end{tabular}

Holidays dataset, $\overleftrightarrow{\xi}$ test gives $\tau=0.75$, while $\vec{\xi}$ test returns $\tau$ equal to $0.49,0.43,0.50$ respectively.

Regarding the matching results, BRIGHT $^{+}$achieves the best performance on the $[128,162]$ size range, with the best mAP values for $\overleftrightarrow{\xi_{\alpha}}$ equal to 0.642 and 0.404 on Holidays and Oxford respectively. On 256 bit class descriptors, OGB achieves an impressive 0.710 on Holidays and 0.520 on Oxford. Then, CDVS-TM 256 achieves 0.679 and 0.437 respectively. In $[486,512]$ size range SVZ gets 0.728 and 0.517 , while BAMBrisk 5120.703 for Holidays and LDB 0.549 for Oxford. Best non binary descriptors are SIFT for $\overleftrightarrow{\xi_{\alpha}}$ test $(0.706$ and 0.488$)$ and LQ-HoG for $\overrightarrow{\xi_{\alpha}}$ test $(0.702$ and 0.466$)$. A comparison between different mAP trends by varying matching tests and descriptors is shown in Figure 6.

Regarding the impact of keypoint detection on matching performance, Table IV shows a comparison for $\overleftrightarrow{\xi_{\alpha}}$ test by means of Hessian-Affine and BRISK detectors on Holidays and Oxford datasets. On average, Hessian-Affine regions improves mAP results by $10 \%$ to $20 \%$ on Holidays and $5 \%$ to $9 \%$ on Oxford. Matching performance can be improved at the cost of time performance (see Section V-E). In summary, the choice of keypoint detector, descriptor technique, matching test and ratio threshold has a huge impact on the overall performance in different datasets.

Bit selection procedure: In order to further compress the descriptors, we applied a fast feature selection technique based on conditional mutual information and a naive Bayesian classifier [65]. The input of the algorithm is the required com-
BEST MAP VALUES FOR $\left(\overleftrightarrow{\xi_{\alpha}}, \mu_{1}\right)$ TEST ON DIFFERENT DETECTORS.

\begin{tabular}{|c|c|c|c|c|c|c|}
\hline \multirow{2}{*}{ Descriptor } & \multicolumn{3}{|c|}{ Holidays dataset [43] } & \multicolumn{3}{|c|}{ Oxford dataset [44] } \\
\hline & HessAff & BRISK & Diff & HessAff & BRISK & Diff \\
\hline BAMBrisk $_{128}$ & 0.603 & 0.455 & 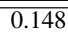 & 0.252 & 0.237 & $\overline{0.015}$ \\
\hline BRIGHT & 0.631 & 0.546 & 0.085 & 0.388 & 0.319 & 0.069 \\
\hline CDVS-TM $_{160}$ & 0.639 & 0.527 & 0.111 & 0.397 & 0.342 & 0.055 \\
\hline BRIGHT $^{+}$ & 0.642 & 0.566 & 0.076 & 0.404 & 0.340 & 0.064 \\
\hline BRIEF & 0.616 & 0.503 & 0.113 & 0.328 & 0.314 & 0.014 \\
\hline ORB & 0.642 & 0.535 & 0.107 & 0.379 & 0.330 & 0.049 \\
\hline ALOHA & 0.659 & 0.567 & 0.093 & 0.421 & 0.354 & 0.067 \\
\hline $\mathrm{CDVS}_{-} \mathrm{TM}_{256}$ & 0.679 & 0.572 & 0.107 & 0.437 & 0.378 & 0.059 \\
\hline BAMBrisk $_{256}$ & 0.669 & 0.537 & 0.132 & 0.314 & 0.305 & 0.008 \\
\hline BinBoost & 0.663 & 0.560 & 0.103 & 0.386 & 0.352 & 0.034 \\
\hline $\mathrm{LATCH}_{256}$ & 0.668 & 0.560 & 0.108 & 0.388 & 0.342 & 0.047 \\
\hline OGB & 0.710 & 0.655 & 0.055 & 0.520 & 0.469 & 0.051 \\
\hline MOBIL & 0.650 & 0.570 & 0.080 & 0.413 & 0.351 & 0.062 \\
\hline LDB & 0.672 & 0.611 & 0.061 & 0.459 & 0.380 & 0.080 \\
\hline FREAK & 0.584 & 0.396 & 0.188 & 0.274 & 0.214 & 0.061 \\
\hline BRISK & 0.684 & 0.552 & 0.132 & 0.424 & 0.339 & 0.085 \\
\hline FRIF & 0.498 & 0.204 & 0.294 & 0.141 & 0.122 & 0.020 \\
\hline BAMBrisk $_{512}$ & 0.703 & 0.574 & 0.129 & 0.344 & 0.333 & 0.011 \\
\hline $\mathrm{LATCH}_{512}$ & 0.696 & 0.619 & 0.077 & 0.432 & 0.376 & 0.056 \\
\hline SVZ & 0.728 & 0.657 & 0.071 & 0.517 & 0.447 & 0.070 \\
\hline
\end{tabular}

pressed descriptor size $\tilde{n}_{b}$ and a list of XOR bit-wise strings computed on the matching and non-matching descriptor pairs from CDVS dataset. The algorithm returns the $\tilde{n}_{b}$ indexes of the selected dimensions. Table V shows mAP values obtained with the compressed descriptors on $\vec{\xi}_{\alpha}$ test without normalization. We also provide average matching results obtained by selecting bit subsets in a random fashion (average over 3 runs). The algorithm brings modest improvements over the random selection for LDB and BRISK descriptors, and reduces 
TABLE III

BEST MEAN AVERAGE PRECISION (MAP) RESULTS FOR DIFFERENT DATASETS AND MATCHING TESTS ON HESSIAN-AFFINE KEYPOINTS $(\mu=1)$.

\begin{tabular}{|c|c|c|c|c|c|c|c|c|c|c|c|c|c|c|c|c|}
\hline \multirow{3}{*}{ Descriptor } & \multicolumn{8}{|c|}{ Holidays dataset [43] } & \multicolumn{8}{|c|}{ Oxford dataset [44] } \\
\hline & \multicolumn{2}{|c|}{$\overleftrightarrow{\xi_{\alpha}}$} & \multicolumn{2}{|c|}{$\overrightarrow{\xi_{\alpha}}$} & \multicolumn{2}{|c|}{$\overleftrightarrow{\xi_{h}}$} & \multicolumn{2}{|c|}{$\overrightarrow{\xi_{h}}$} & \multicolumn{2}{|c|}{$\overleftrightarrow{\xi_{\alpha}}$} & \multicolumn{2}{|c|}{$\overrightarrow{\xi_{\alpha}}$} & \multicolumn{2}{|c|}{$\overleftrightarrow{\xi_{h}}$} & \multicolumn{2}{|c|}{$\overrightarrow{\xi_{h}}$} \\
\hline & $\tau$ & mAP & $\tau$ & $\mathrm{mAP}$ & $h^{*}$ & mAP & $h^{*}$ & mAP & $\tau$ & $\mathrm{mAP}$ & $\tau$ & mAP & $h^{*}$ & $\mathrm{mAP}$ & $h^{*}$ & $\mathrm{mAP}$ \\
\hline BAMBrisk $_{128}$ & 0.68 & 0.603 & $\mathbf{0 . 5 0}$ & 0.550 & $\overline{0.07}$ & 0.516 & 0.07 & 0.511 & 0.72 & 0.252 & 0.67 & 0.207 & 0.10 & 0.105 & 0.10 & 0.105 \\
\hline BRIGHT & 0.71 & 0.631 & 0.53 & 0.534 & 0.15 & 0.627 & 0.16 & 0.620 & 0.81 & 0.388 & 0.76 & 0.276 & 0.18 & 0.213 & 0.23 & 0.196 \\
\hline CDVS-TM $_{160}$ & 0.67 & 0.639 & 0.45 & 0.515 & 0.08 & 0.486 & 0.08 & 0.480 & 0.79 & 0.397 & 0.75 & 0.284 & 0.12 & 0.184 & 0.12 & 0.179 \\
\hline BRIGHT $^{+}$ & 0.73 & 0.642 & 0.55 & 0.537 & 0.15 & 0.637 & 0.16 & 0.625 & 0.82 & 0.404 & 0.75 & 0.286 & 0.22 & 0.230 & 0.20 & 0.207 \\
\hline BRIEF & 0.67 & 0.616 & 0.30 & 0.368 & 0.05 & 0.496 & 0.05 & 0.489 & 0.74 & 0.328 & 0.55 & 0.193 & 0.07 & 0.134 & 0.08 & 0.128 \\
\hline ORB & 0.73 & 0.642 & 0.36 & 0.359 & 0.09 & 0.538 & 0.09 & 0.532 & 0.78 & 0.379 & 0.59 & 0.223 & 0.09 & 0.158 & 0.08 & 0.152 \\
\hline ALOHA & 0.70 & 0.659 & 0.54 & 0.572 & 0.13 & 0.586 & 0.14 & 0.581 & 0.73 & 0.421 & 0.65 & 0.305 & 0.15 & 0.301 & 0.14 & 0.293 \\
\hline CDVS-TM $_{256}$ & 0.75 & 0.679 & 0.50 & 0.550 & 0.10 & 0.530 & 0.09 & 0.526 & 0.80 & 0.437 & 0.75 & 0.298 & 0.20 & 0.228 & 0.15 & 0.215 \\
\hline BAMBrisk $_{256}$ & 0.74 & 0.669 & 0.46 & 0.536 & 0.07 & 0.562 & 0.07 & 0.557 & 0.77 & 0.314 & 0.65 & 0.230 & 0.08 & 0.116 & 0.08 & 0.117 \\
\hline $\mathrm{BinB}$ & 0.75 & 0.663 & 0.45 & 0.458 & 0.10 & 0.599 & 0.11 & 0.588 & 0.82 & 0.386 & 0.75 & 0.240 & 0.26 & 0.182 & 0.30 & 0.156 \\
\hline $\mathrm{LATCH}_{256}$ & 0.75 & 0.668 & 0.43 & 0.450 & 0.11 & 0.492 & 0.10 & 0.488 & 0.79 & 0.388 & 0.69 & 0.289 & 0.14 & 0.160 & 0.13 & 0.158 \\
\hline OGB & 0.81 & 0.710 & 0.62 & 0.596 & 0.10 & 0.543 & 0.11 & 0.519 & 0.89 & 0.520 & 0.80 & 0.299 & 0.25 & 0.364 & 0.15 & 0.205 \\
\hline MOBIL & 0.68 & 0.650 & 0.38 & 0.507 & 0.08 & 0.613 & 0.08 & 0.616 & 0.75 & 0.413 & 0.57 & 0.289 & 0.08 & 0.257 & 0.09 & 0.256 \\
\hline LDB & 0.70 & 0.672 & 0.41 & 0.541 & 0.09 & 0.609 & 0.09 & 0.601 & 0.76 & 0.459 & 0.60 & 0.321 & 0.10 & 0.290 & 0.10 & 0.279 \\
\hline FREAK & 0.64 & 0.584 & 0.36 & 0.329 & 0.04 & 0.416 & 0.04 & 0.414 & 0.73 & 0.274 & 0.55 & 0.153 & 0.07 & 0.101 & 0.07 & 0.101 \\
\hline BRISK & 0.75 & 0.684 & 0.49 & 0.519 & 0.09 & 0.598 & 0.09 & 0.594 & 0.74 & 0.424 & 0.61 & 0.285 & 0.11 & 0.189 & 0.11 & 0.181 \\
\hline FRIF & 0.75 & 0.498 & 0.43 & 0.256 & 0.06 & 0.226 & 0.06 & 0.227 & 0.77 & 0.141 & 0.56 & 0.078 & 0.50 & 0.079 & 0.46 & 0.079 \\
\hline BAMBrisk $_{512}$ & 0.75 & 0.703 & 0.50 & 0.571 & 0.07 & 0.590 & 0.07 & 0.577 & 0.75 & 0.344 & 0.62 & 0.239 & 0.09 & 0.124 & 0.09 & 0.123 \\
\hline LATC & 0.78 & 0.696 & 0.44 & 0.475 & 0.12 & 0.551 & 0.12 & 0.547 & 0.78 & 0.432 & 0.63 & 0.331 & 0.35 & 0.193 & 0.13 & 0.180 \\
\hline SVZ & 0.79 & 0.728 & 0.52 & 0.581 & 0.11 & 0.678 & 0.14 & 0.671 & 0.87 & 0.517 & 0.80 & 0.356 & 0.22 & 0.326 & 0.22 & 0.324 \\
\hline SURF & 0.53 & 0.638 & $\overline{0.1}$ & & 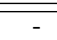 & - & - & & 0.64 & 0.383 & 0.3 & & & & - & - \\
\hline SIFT & & & 0.35 & & - & - & - & - & 0.73 & 0.488 & 0.57 & & - & - & - & - \\
\hline LQ-HoG & 0.67 & 702 & 0.44 & & - & - & - & - & 0.71 & 0.466 & 0.59 & 0.413 & - & - & - & - \\
\hline LIOP & 0.77 & 0.665 & 0.32 & 0.481 & - & - & - & - & 0.70 & 0.363 & 0.57 & 0.193 & - & - & - & - \\
\hline MROGH & 0.74 & 0.672 & 0.30 & 0.420 & - & - & - & - & 0.75 & 0.380 & 0.55 & 0.159 & - & - & - & - \\
\hline MRRID & 0.77 & 0.618 & 0.40 & 0.357 & - & - & - & - & 0.77 & 0.340 & 0.59 & 0.146 & - & - & - & - \\
\hline
\end{tabular}

TABLE V

Bit SELECTION $\left(\overleftrightarrow{\xi_{\alpha}}, \mu_{1}\right)$ TRAINED WITH CDVS PATCHES DATASET ON HESSIAN-AFFINE KEYPOINTS.

\begin{tabular}{|l||c||c|c||c|c|}
\hline \multicolumn{1}{|l||}{ Descriptor } & \multirow{2}{*}{ mAP } & \multicolumn{2}{|c||}{$\begin{array}{c}\tilde{n}_{b}=256 \\
\text { Select / Rand }\end{array}$} & \multicolumn{2}{|c|}{$\begin{array}{c}\tilde{n}_{b}=128 \\
\text { Select / Rand }\end{array}$} \\
\hline \hline Holidays dataset & & & & & \\
ALOHA & 0.659 & - & - & $\mathbf{0 . 6 1 8}$ & 0.590 \\
BinBoost & 0.663 & - & - & 0.580 & 0.582 \\
BRIGHT $^{+}$ & 0.642 & - & - & $\mathbf{0 . 6 3 2}$ & 0.620 \\
MOBIL $^{\text {LDB }}$ & 0.650 & $\mathbf{0 . 6 4 7}$ & 0.644 & 0.587 & 0.594 \\
BRISK & 0.672 & $\mathbf{0 . 6 5 4}$ & 0.643 & $\mathbf{0 . 5 8 1}$ & 0.568 \\
BAMBrisk 512 & 0.684 & $\mathbf{0 . 6 2 1}$ & 0.601 & $\mathbf{0 . 5 1 6}$ & 0.486 \\
\hline Oxford dataset & 0.703 & 0.596 & 0.652 & 0.424 & 0.571 \\
ALOHA & & & & & \\
BinBoost & 0.421 & - & - & $\mathbf{0 . 3 7 9}$ & 0.344 \\
BRIGHT & 0.386 & - & - & 0.292 & 0.293 \\
MOBIL & 0.404 & - & - & $\mathbf{0 . 3 8 4}$ & 0.356 \\
LDB & 0.413 & $\mathbf{0 . 4 0 9}$ & 0.406 & 0.338 & 0.343 \\
BRISK & 0.459 & $\mathbf{0 . 4 2 5}$ & 0.412 & $\mathbf{0 . 3 3 9}$ & 0.329 \\
BAMBrisk & 0.424 & $\mathbf{0 . 3 4 6}$ & 0.322 & $\mathbf{0 . 2 5 8}$ & 0.238 \\
BA12 & 0.344 & 0.245 & 0.300 & 0.157 & 0.225 \\
\hline
\end{tabular}

performance for BAMBrisk 512 , which is already highly optimized using a different feature selection mechanism. Improved performance are highlighted in the table.

\section{Image-level matching performance}

Dataset [45] has been used in many works [11], [12], [16], [20] to assess the performance of descriptors by applying several transformations on the images such as zoom, rotation, viewpoint change, blur, compression and light change. For each transformation, six images sorted according to ascending difficulty and ground-truth homography between the first image and the others are provided. The corresponding keypoints, already provided in the dataset, are identified by analyzing the overlap area of the Hessian-Affine region in one image and the projection of the region in a second image In this test, a region is considered correspondent if its intersection is larger than $50 \%$ of the union of the two regions. Figure 7 shows the TPR values for each transformation by using an average ratio threshold $\bar{\tau}$ on the best ones from Holidays and Oxford dataset. The $\overrightarrow{\xi_{1}}$ test was used to be consistent with previous evaluations. Since, apart from bark sequence, ROC curves show a monotonic descending trend according to the ascending difficulty, transformation $(1 \rightarrow 4)$ was chosen to comment the results. FPR values for all the descriptors are zero or very low (e.g. $\leq 0.003$ on $u b c$ ), therefore they can be fairly compared. Since OGB works in different (FPR, TPR) ranges, its curves are omitted. Zoom and rotation transformations show different results: on bark the best descriptors are BAMBrisk (0.138 0.114 ) and SVZ (0.098), while on boat LDB (0.054) and SVZ (0.082). For image blur, LDB (0.074), BRIGHT $^{+}(0.066)$ and ALOHA (0.056) are selected, for viewpoint change and light change SVZ $(0.051,0.145)$, BRIGHT $^{+}(0.037,0.114)$ and CDVS-TM ${ }_{256}(0.037,0.141)$, for JPEG compression LDB (0.379), ALOHA (0.310), SVZ (0.293) and BAMBrisk 512 $(0.276)$.

Table VI summarizes the matching performance for all the proposed transformations by means of an aggregate metric $\bar{p}_{\delta}$. For each transformation sequence $T$, descriptor technique $\delta$ and transformation $(1 \rightarrow q)$, the Euclidean distance $e_{\delta, q}^{(T)}$ between (FPR, 1 - TPR) values and the origin is computed. 


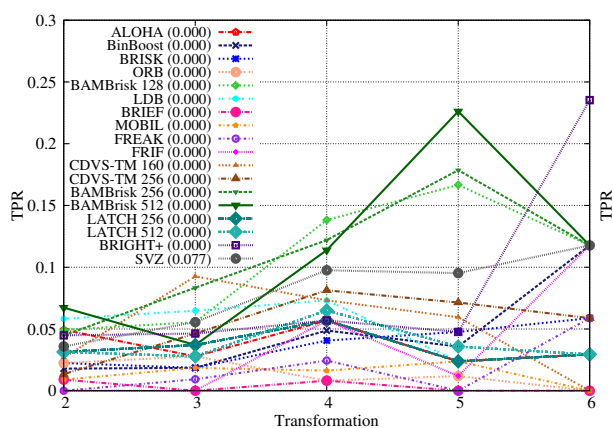

(a) Zoom + rotation (bark)

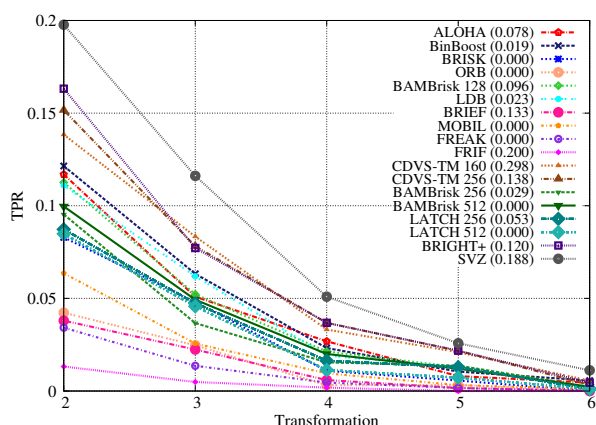

(d) Viewpoint change (graf)

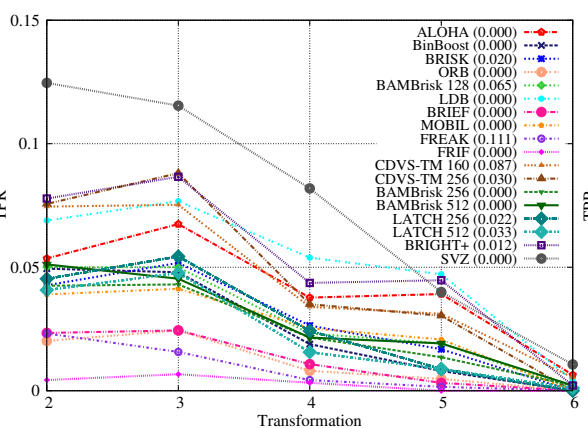

(b) Zoom + rotation (boat)

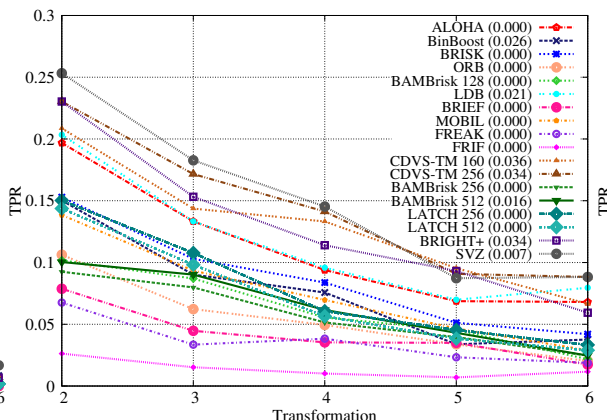

(e) Light change (leuven)

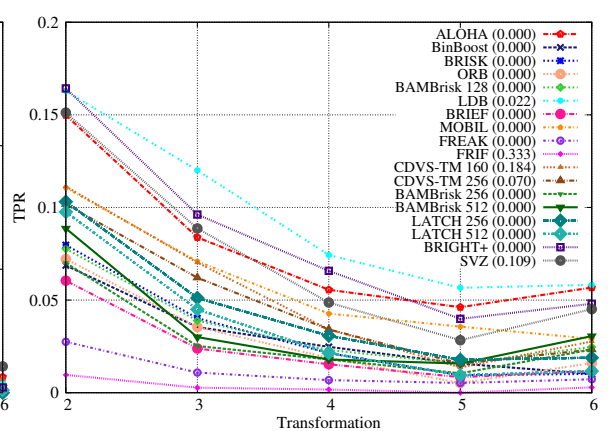

(c) Image blur (bikes)

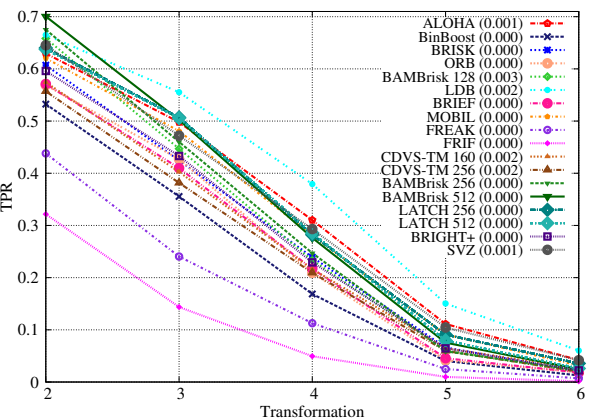

(f) JPEG compression (ubc)

Fig. 7. Comparison of TPR values for different binary descriptors extracted using the Hessian-Affine detector on Mikolajczyk dataset [45]: (a) and (b) zoom with rotation, (c) image blur, (d) viewpoint change, (e) light change, (f) JPEG compression. Transformations $(1 \rightarrow 2),(1 \rightarrow 3) \ldots(1 \rightarrow 6)$ are sorted according to ascending difficulty. On the legend, FPR values corresponding to the chosen ratio threshold $\tau$ for transformation $(1 \rightarrow 4)$ are in parentheses.

TABLE VI

OVERALl IMAGE-LEVEL PERFORMANCE: BeSt $(\bullet)$ AND WORST $(\times)$ CASE.

\begin{tabular}{|l||c||c|c|c|c|c|c|}
\hline Descriptor & $\bar{p}_{\delta}$ & bar & boa & bik & gra & leu & ubc \\
\hline \hline BAMBrisk 128 & $\mathbf{9 1 . 0 1}$ & $\bullet$ & & & & $\times$ & \\
BRIGHT & 88.72 & & & & $\bullet$ & & $\times$ \\
CDVS-TM 160 & 81.60 & & & & $\bullet$ & & $\times$ \\
BRIGHT+ & 88.83 & $\bullet$ & & & & & $\times$ \\
\hline BRIEF & 79.90 & & & & $\bullet$ & $\times$ & \\
ORB & 79.11 & & & & $\bullet$ & $\times$ & \\
ALOHA & 87.66 & & & & $\bullet$ & $\times$ & \\
CDVS-TM 256 & 85.69 & $\bullet$ & & & & & $\times$ \\
BAMBrisk 256 & 91.64 & $\bullet$ & & & & $\times$ & \\
BinBoost & 87.63 & & & & $\bullet$ & & $\times$ \\
LATCH 256 & 84.67 & $\bullet$ & & & & $\times$ & \\
OGB & $\mathbf{1 0 0 . 0 0}$ & & & & $\bullet$ & $\times$ & \\
MOBIL & 84.30 & & & & $\bullet$ & \\
\hline LDB & 85.84 & $\bullet$ & & & & $\times$ & \\
FREAK & 78.83 & & & & $\bullet$ & & $\times$ \\
BRISK & 84.76 & $\bullet$ & & & & $\times$ & \\
FRIF & 74.46 & $\bullet$ & & & & $\times$ & \\
BAMBrisk 512 & $\mathbf{9 2 . 1 5}$ & $\bullet$ & & & $\bullet$ & $\times$ & \\
LATCH 512 & 87.16 & & & & & & $\times$ \\
SVZ & 88.62 & $\bullet$ & & & & \\
\hline
\end{tabular}

The lowest value $e_{\min , q}^{(T)}$ for each $T$ is compared to the actual value for each $\delta$ and a percentage $p_{\delta, q}^{(T)}$ is computed:

$$
p_{\delta, q}^{(T)}=\frac{e_{m i n, q}^{(T)} \cdot 100}{e_{\delta, q}^{(T)}}
$$

Then, for each $\delta$ the mean value $\bar{p}_{\delta}^{(T)}$ among the $q$ transformations is taken. The worst value represents the aggregate metric:

$$
\bar{p}_{\delta}=\min _{T} \bar{p}_{\delta}^{(T)}
$$

OGB shows the best performance with an impressive $100 \%$, while the BAMBrisk family is always above 90\%. Light change (leuven) and JPEG compression $(u b c)$ are the most difficult sequences to deal with, while zoom+rotation (bark) and viewpoint change (graf) are the easiest.

Image-level datasets are very useful in evaluating robustness of descriptors to individual transformations, but they also present some issues, since distortions and geometric transformations are generated artificially. Number of matching descriptor pairs is also limited and, more importantly, the number of distractors is relatively small. In the next paragraph a challenging video dataset for mobile applications is proposed to better understand the overall impact of different transformations on the matching performance of the descriptors.

\section{Mobile dataset matching performance}

We propose a new dataset for objects retrieval on mobile scenario. The dataset consists of 8658 pictures extracted from video sequences shooting a table containing several objects, including postcards, little boxes, papers, flowers, glasses, bottles, business cards and magazines. Three different devices were used: GoPro 3, Nexus 7, and iPhone 5s. Images were collected from video at rate of 5 frames per second. As shown in Figure 8, six query objects containing features extracted using the Hessian-Affine detector have been selected: Paper, 


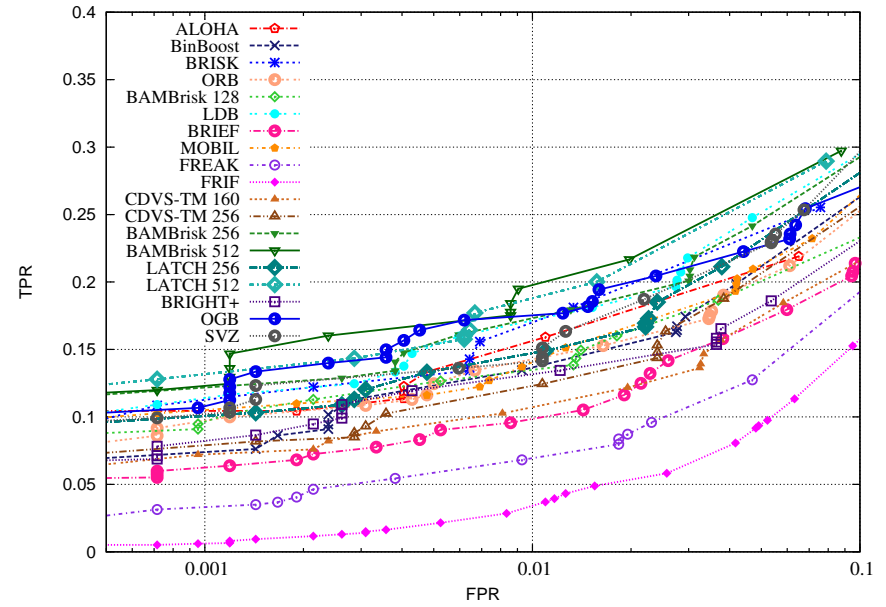

(a) Postcard query

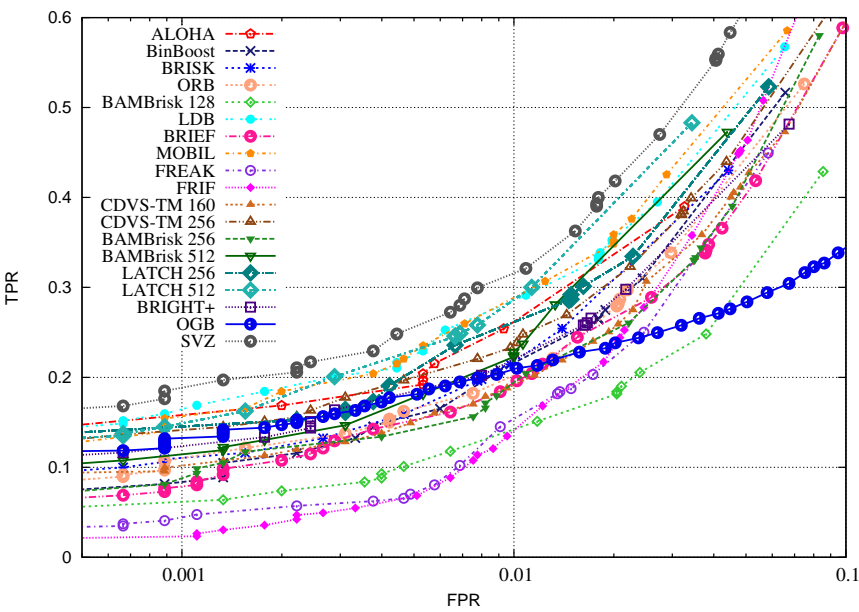

(c) Magazine query

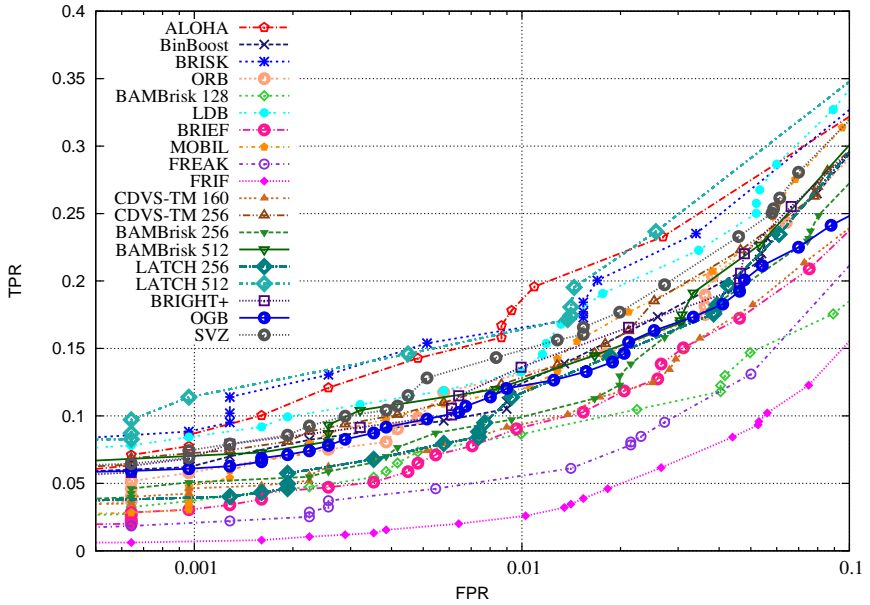

(b) Blue box query

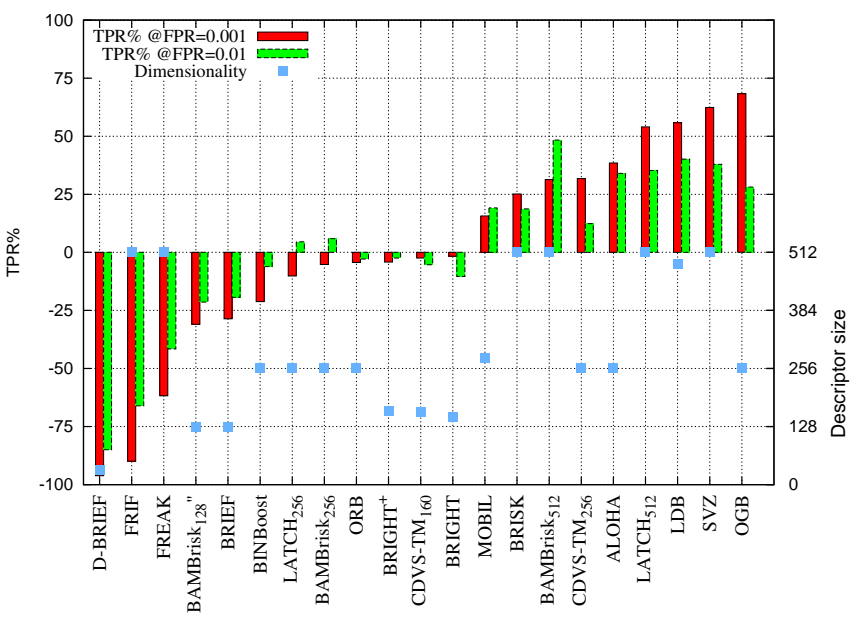

(d) Overall performance

Fig. 9. Comparison of ROC curves for three queries on the proposed mobile dataset: (a) Postcard, (b) Blue box and (c) Magazine. Matching test considered is $\overleftrightarrow{\xi_{\alpha}}$ with $\mu_{1}$ normalization and ratio threshold $\bar{\tau}$ as mean value between the optimum thresholds computed for Holidays and Oxford datasets using the Hessian-Affine detector. (d) Overall variation in percentage respect to average TPR value at operating point FPR $=0.01$ and FPR $=0.001$.
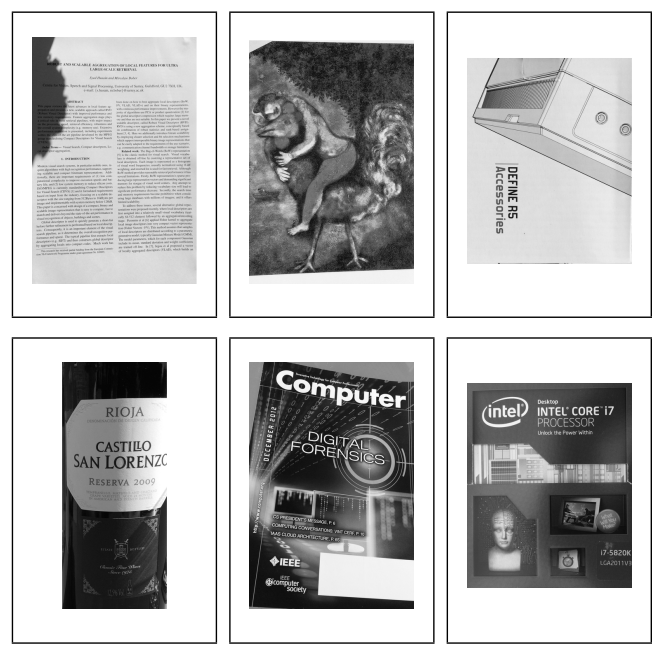

Fig. 8. Queries used in the proposed mobile dataset. From left to right and top to bottom, with number of Hessian-Affine keypoints in parentheses: Paper (15848), Postcard (3308), White box (3144), Wine (2843), Magazine (18912) and Blue box (9374).
Postcard, White box, Wine, Magazine and Blue box. The dataset is available at [62].

Figure 9 shows the ROC performance for binary descriptors on three query objects by means of $\overleftrightarrow{\xi_{\alpha}}$ matching test, $\mu_{1}$ normalization and ratio threshold $\bar{\tau}$ as mean value between the optimum thresholds computed for Holidays and Oxford datasets. An object is detected in an incoming query image if $\alpha\left(d_{a b}\right) \geq \alpha^{*}$, where $d_{a b}$ are the descriptors which passed the $\overleftrightarrow{\xi_{\alpha}}$ matching test and $\alpha^{*}$ is the threshold. While for Postcard, Magazine and Blue box descriptors show different behavior according to their intrinsic effectiveness, the other queries do not prove any detection capability. For instance, White box and Wine lack in distinctive features, and the surface is almost plain color. Moreover, these objects include several alphabetical letters, easily causing false positive matches because of their geometric characteristics. Paper contains many features, but these can be detected with a high level of zoom only, therefore the object may be present in a frame, but no matching features can be found. By considering FPR $=0.01$, on Postcard query the best descriptors are BAMBrisk ${ }_{512}$ (TPR 
TABLE VII

TIMING RESULTS FOR DESCRIPTORS EXTRACTION. CDVS-TM (*) TIMING DOES NOT INCLUDE SIFT PRE-COMPUTATION.

\begin{tabular}{|c|c|c|c|c|c|}
\hline Descriptor & $\begin{array}{r}\Delta \\
\mathrm{PC} /\end{array}$ & $\begin{array}{l}\text { ms) } \\
\text { ndroid }\end{array}$ & Descriptor & $\begin{array}{r}\Delta \\
\mathrm{PC} /\end{array}$ & $\begin{array}{l}\text { ms) } \\
\text { ndroid }\end{array}$ \\
\hline MRRID & 6.97 & 18.69 & ALOHA & 0.07 & 0.20 \\
\hline MROGH & 4.55 & 11.97 & MOBIL & 0.05 & 0.31 \\
\hline SVZ & 3.02 & - & FRIF & 0.04 & 0.14 \\
\hline BinBoost & 2.30 & 5.02 & ORB & 0.03 & 0.31 \\
\hline LIOP & 1.34 & 2.65 & SURF & 0.03 & 0.11 \\
\hline $\mathrm{LATCH}_{512}$ & 0.71 & 1.08 & D-BRIEF & 0.03 & 0.09 \\
\hline $\mathrm{LATCH}_{256}$ & 0.36 & 0.55 & BRIEF & 0.03 & 0.07 \\
\hline SIFT & 0.23 & 1.88 & BAMBrisk $_{128}$ & 0.03 & 0.07 \\
\hline LQ-HoG & 0.22 & 1.54 & BAMBrisk $_{256}$ & 0.03 & 0.07 \\
\hline OGB & 0.21 & 1.08 & BAMBrisk $_{512}$ & 0.03 & 0.07 \\
\hline BRIGHT $^{+}$ & 0.21 & 0.73 & BRISK & 0.01 & 0.04 \\
\hline BRIGHT & 0.19 & 0.69 & FREAK & 0.01 & 0.03 \\
\hline BRIGHT $^{o p t}$ & 0.16 & 0.68 & CDVS-TM ${ }_{256}$ & $0.01^{*}$ & $0.01^{*}$ \\
\hline LDB & 0.12 & 0.47 & CDVS-TM $_{160}$ & $0.01^{*}$ & $0.01 *$ \\
\hline
\end{tabular}

$=0.199), \mathrm{LATCH}_{512}(0.189)$, OGB (0.174) and LDB (0.170). On Magazine query, the best descriptors are SVZ (0.316), $\mathrm{LATCH}_{512}(0.285)$, LDB (0.284) and MOBIL (0.282). On Blue box query, the best descriptors are ALOHA (0.188), $\mathrm{LATCH}_{512}$ (0.163) and BRISK (0.163). Figure 9d summarizes the overall matching performance by considering an aggregate metric TPR\% at different FPR values. For each query $q$, the average TPR value among all the descriptors is defined as $\mathrm{TPR}_{q}$, then for each descriptor $\delta$ the difference in percentage from the average is used as matching index. This index is averaged on all the queries to get the final TPR\% metric:

$$
T P R_{\delta} \%=\underset{q}{\operatorname{avg}}\left\{\frac{T P R_{\delta}-T P R_{q}}{T P R_{q}} \cdot 100\right\} .
$$

The following descriptors show ascending matching performance over the average at FPR $=0.001$ : BRISK $(+25.1 \%)$, BAMBrisk $_{512}(+31.4 \%)$, CDVS-TM $256(+31.7 \%)$, ALOHA $(+38.5 \%), \quad$ LATCH $_{512}(+54.0 \%), \quad$ LDB $(+55.8 \%), \quad$ SVZ $(+62.4 \%)$ and OGB $(68.3 \%)$.

\section{E. Keypoint detection and descriptor extraction complexity}

In this section we analyze the complexity of keypoint detection and descriptor computation. Table VII shows the average time for a single descriptor extraction on a DELL PowerEdge R730 server @3.1GHz and on a HTC ONE M8 smartphone with Qualcomm MSM8974AB Snapdragon 801 $@ 2.3 \mathrm{GHz}$ by using a single core. Descriptors are extracted from CDVS patches dataset. The source code for all the algorithms was compiled on the above mentioned architecture, with no use of any pre-compiled executable. A timestamp was introduced at the beginning and at the end of the descriptor extraction routine, and time difference was divided by the total number of patches. OpenCV v2.4.8 with Java Native Interface (JNI) was used for the Android device. A new version of BRIGHT, called BRIGHT ${ }^{\text {opt }}$, computes gradient orientation histograms on the full patch only instead of taking each patch partition separately. This operation avoids border effects and makes the processing faster. BinBoost is the slowest binary descriptor, while BRISK is 230 times faster. As expected, descriptors requiring the computation of gradient information are remarkably slower than methods requiring pixel intensities only. In summary, ALOHA, LDB, MOBIL, ORB, FRIF, DBRIEF, BRIEF, BAMBrisk, FREAK and BRISK return a descriptor in less than $0.5 \mathrm{~ms}$ for both the architectures.

For the analysis of the keypoint detection complexity, we compute the average number of keypoints $\bar{n}_{k}$ and the average time $\bar{t}$ for keypoints extraction per image for Hessian-Affine and BRISK detectors on Oxford and Holidays datasets. On Oxford $\bar{n}_{k}=13588$ and $\bar{t}=1350 \mathrm{~ms}$ for Hessian-Affine, while $\bar{n}_{k}=8514$ and $\bar{t}=85 \mathrm{~ms}$ for BRISK. On Holidays $\bar{n}_{k}=17471$ and $\bar{t}=1200 \mathrm{~ms}$ for Hessian-Affine, while $\bar{n}_{k}=$ 9254 and $\bar{t}=85 \mathrm{~ms}$ for BRISK. Therefore, on image basis, BRISK detector is 14 to 16 times faster than Hessian-Affine.

\section{F. Critical analysis}

As shown in the previous sections, the ranking of the descriptors' matching capabilities may vary depending on the evaluation protocol and the associated dataset. Patch-level evaluation gives a coarse assessment of the discriminative power using pre-extracted regions around the keypoints. These datasets are also often used for training using machine learning techniques. Database-level evaluation better reflects real-life scenarios such as image retrieval, while the proposed mobile dataset provides the ultimate challenge due to its complexity.

Taking into account all the results achieved, BAMBrisk and BRISK represent the best trade-off between matching capabilities and complexity, proving to be robust in database retrieval under all the proposed geometric transformations. Their simple designs based on intensity comparison patterns compete well against CPU-demanding descriptors based on gradient histograms. Regarding other intensity-only descriptors, the well-known ORB, BRIEF and FREAK lack in matching capabilities at low false positive rates. The first two use individual sampling points, which are highly sensitive to noise within the patch. This problem is also present in FRIF and explains its poor results. FREAK makes use of sampling regions instead, but the deeply overlapping patterns imply the coding of redundant information. ALOHA delivers average performance in the mobile scenario. Nevertheless, the intuitive multi-layer sampling pattern can be an inspiration for new low-complexity designs. LATCH triplets comparisons are also effective, since they mitigate noise and local appearance variations, but definitely slower then the previously mentioned methods. The D-BRIEF projections and thresholding operations are extremely fast to perform, but the descriptor size is simply too compact to show any effectiveness in large-scale retrieval.

Regarding the descriptors using gradient information, OGB, LDB and BRIGHT proved to be the most accurate. OGB is slow to extract, however it can be considered the undisputed winner in all the test scenarios. Its bilinear interpolation distributes the value of each gradient sample into adjacent histogram bins, contributing in the composition of a very robust compaction method. LDB features, combining intensity and gradient information, capture distinct patterns of the patch at different spatial granularities. They have proved to be very robust especially in the mobile scenario, where specific 
distortions in illumination or scaling occur simultaneously. A note of merit for BRIGHT: the block scan ordering and the progressive bit selection result in a highly discriminative descriptor, in both patch-level and database-level tests, even with only 150 dimensions. MOBIL embeds a different type of second-order intensity information, the moments, delivering average performance. More studies on its comparison patterns are needed to improve the performance, but this comes at cost of extraction complexity.

CDVS-TM and SVZ must be considered separately because they are not natively binary. CDVS-TM requires a dedicated look-up table for the computation of the Hamming distance. For this reason, the main benefit of using binary descriptors is jeopardized. SVZ requires a binarization procedure of realvalued descriptors and the storage of massive pre-trained matrices, which are prohibitive for an embedded scenario. BinBoost is also slow in extraction, however the overall results prove that the matching performance does not depend on the datasets used in the training stage.

\section{CONCLUSIONS}

In this paper we presented a comprehensive, in-depth evaluation of binary descriptors for mobile applications. We enhanced existing evaluation protocols with a new one, including challenging video test sequences. They reflect the typical mobile scenarios, where poor illumination, motion blur and coding artifacts are often present. The performance measures we designed are also better aligned with the ultimate goal - recognition of objects seen in a camera of mobile device. The evaluation was performed using the fast BRISK detector, with the full Hessian-Affine acting as benchmark reference. The discriminative power of 21 binary descriptors was tested, showing that gradient-based solutions such as LATCH, OGB and LDB all perform well at cost of more demanding processing support. Additionally, we also evaluated the MPEGstandardized descriptor (CDVS-TM) and the state-of-the-art SVZ descriptor to provide performance context, even though the extraction time is prohibitive for embedded systems.

Nevertheless, the overall matching performance of descriptors such as ALOHA, BAMBrisk and BRISK demonstrates the feasibility of competitive designs based on binary intensity tests only, which proved to be robust to different geometric transformations and illumination changes. Intensity tests are also extremely efficient when applied on integral images, thus well suited for mobile applications. Therefore, future designs should explore in depth sub-patch intensity patterns and dimensionality reduction tools in order to build superfast, discriminative and compact binary descriptors.

We also note that the feature detectors occasionally fail to find keypoints consistently in down-sampled images, affecting both extraction and matching of distinctive descriptors. Future research should also address this area.

\section{REFERENCES}

[1] V. Chandrasekhar, G. Takacs, D. Chen, S. Tsai, Y. Reznik, R. Grzeszczuk, and B. Girod, "Compressed histogram of gradients: A low-bitrate descriptor," International Journal of Computer Vision, vol. 96, no. 3, pp. 384-399, 2012.
[2] J. Eakins, M. Graham, and T. Franklin, "Content-based image retrieval," Library and Information Briefings, vol. 85, pp. 1-15, 1999.

[3] W. Tan, B. Yan, K. Li, and Q. Tian, "Image retargeting for preserving robust local feature: Application to mobile visual search," IEEE Trans. on Multimedia, vol. 18, no. 1, pp. 128-137, Jan 2016.

[4] L. Y. Duan, R. Ji, Z. Chen, T. Huang, and W. Gao, "Towards mobile document image retrieval for digital library," IEEE Trans. on Multimedia, vol. 16, no. 2, pp. 346-359, Feb 2014.

[5] H. Qi, M. Stojmenovic, K. Li, Z. Li, and W. Qu, "A low transmission overhead framework of mobile visual search based on vocabulary decomposition," IEEE Trans. on Multimedia, vol. 16, no. 7, pp. 19631972, Nov 2014.

[6] E. Mair, G. D. Hager, D. Burschka, M. Suppa, and G. Hirzinger, "Adaptive and generic corner detection based on the accelerated segment test," in Computer Vision - ECCV 2010, K. Daniilidis, P. Maragos, and N. Paragios, Eds. Berlin, Heidelberg: Springer, 2010, pp. 183-196.

[7] L. Chen, D. Xu, I. W. Tsang, and J. Luo, "Tag-based image retrieval improved by augmented features and group-based refinement," IEEE Trans. on Multimedia, vol. 14, no. 4, pp. 1057-1067, Aug. 2012.

[8] L. Y. Duan, J. Lin, Z. Wang, T. Huang, and W. Gao, "Weighted component hashing of binary aggregated descriptors for fast visual search," IEEE Trans. on Multimedia, vol. 17, no. 6, pp. 828-842, June 2015.

[9] J. Song, Y. Yang, Z. Huang, H. T. Shen, and J. Luo, "Effective multiple feature hashing for large-scale near-duplicate video retrieval," IEEE Transactions on Multimedia, vol. 15, no. 8, pp. 1997-2008, Dec 2013.

[10] M. Calonder, V. Lepetit, C. Strecha, and P. Fua, "BRIEF: Binary robust independent elementary features," in Proc. of the 11th European Conf. on Comp. Vision, ser. ECCV'10. Springer-Verlag, 2010, pp. 778-792.

[11] S. Leutenegger, M. Chli, and R. Siegwart, "Brisk: Binary robust invariant scalable keypoints," in Computer Vision (ICCV), 2011 IEEE International Conference on, Nov 2011, pp. 2548-2555.

[12] R. Ortiz, "FREAK: Fast retina keypoint," in Proceedings of the 2012 IEEE Conf. on Computer Vision and Pattern Recognition (CVPR). Washington, DC, USA: IEEE Computer Society, 2012, pp. 510-517.

[13] K. Iwamoto, R. Mase, and T. Nomura, "BRIGHT: A scalable and compact binary descriptor for low-latency and high accuracy object identification," in Image Processing (ICIP), 2013 20th IEEE International Conference on, Sept 2013, pp. 2915-2919.

[14] E. Rublee, V. Rabaud, K. Konolige, and G. Bradski, "ORB: An efficient alternative to sift or surf," in Proc. of the 2011 Int. Conf. on Computer Vision, ser. ICCV '11. IEEE Computer Society, 2011, pp. 2564-2571.

[15] T. Trzcinski and V. Lepetit, "Efficient discriminative projections for compact binary descriptors," in Computer Vision - ECCV 2012. Springer Berlin Heidelberg, 2012, vol. 7572, pp. 228-242.

[16] S. Saha and V. Demoulin, "ALOHA: An efficient binary descriptor based on haar features," in Image Processing (ICIP), 2012 19th IEEE International Conference on, Sept 2012, pp. 2345-2348.

[17] G. Levi and T. Hassner, "LATCH: Learned Arrangements of Three Patch Codes," ArXiv e-prints, Jan. 2015.

[18] X. Yang, X. Wang, and K.-T. T. Cheng, "OGB: A distinctive and efficient feature for mobile augmented reality," in MultiMedia Modeling: 22nd International Conference, MMM 2016, Q. Tian, N. Sebe, G.-J. Qi, B. Huet, R. Hong, and X. Liu, Eds.

[19] F. W. Zhenhua Wang, Bin Fan, "FRIF: Fast robust invariant feature," in Proc. of the British Machine Vision Conference. BMVA Press, 2013.

[20] X. Yang and K. Cheng, "LDB: An ultra-fast feature for scalable augmented reality on mobile devices," in Mixed and Augmented Reality (ISMAR), 2012 IEEE Int. Symposium on, Nov 2012, pp. 49-57.

[21] A. Bellarbi, S. Otmane, N. Zenati, and S. Benbelkacem, "[poster] MOBIL: A moments based local binary descriptor," in Mixed and Augmented Reality (ISMAR), 2014 IEEE Int. Symp. on, Sept 2014, pp. 251-252.

[22] L. Baroffio, M. Cesana, A. Redondi, and M. Tagliasacchi, "Bamboo: A fast descriptor based on asymmetric pairwise boosting," in Image Processing (ICIP), 2014 IEEE Int. Conf. on, Oct 2014, pp. 5686-5690.

[23] V. L. T. Trzcinski, M. Christoudias and P. Fua, "Boosting Binary Keypoint Descriptors," in Comp. Vision and Pattern Recognition, 2013.

[24] "Compact descriptors for visual search: Evaluation framework," ISO/IEC JTC1 SC29 WG11 output document N12202, Tech. Rep., July 2011.

[25] H. Bay, A. Ess, T. Tuytelaars, and L. Van Gool, "Speeded-up robust features (SURF)," Comput. Vis. Image Underst., vol. 110, no. 3, pp. 346-359, Jun. 2008.

[26] V. Chandrasekhar, G. Takacs, D. Chen, S. Tsai, M. Makar, and B. Girod, "Feature matching performance of compact descriptors for visual search," in IEEE Data Compression Conference (DCC), 2014. 
[27] Z. Wang, B. Fan, and F. Wu, "Local intensity order pattern for feature description," in In IEEE ICCV, 2011, pp. 603-610.

[28] T. Lindeberg, "Feature detection with automatic scale selection," International Journal of Computer Vision, vol. 30, no. 2, pp. 79-116, 1998

[29] H. Jégou, M. Douze, and C. Schmid, "Exploiting descriptor distances for precise image search," Research Report RR-7656, Jun. 2011 [Online]. Available: https://hal.inria.fr/inria-00602325

[30] S. Zhang, Q. Tian, Q. Huang, W. Gao, and Y. Rui, "USB: Ultrashort binary descriptor for fast visual matching and retrieval," Image Processing, IEEE Transactions on, vol. 23, no. 8, pp. 3671-3683, Aug 2014.

[31] Z. Liu, H. Li, W. Zhou, R. Hong, and Q. Tian, "Uniting keypoints: Local visual information fusion for large-scale image search," IEEE Transactions on Multimedia, vol. 17, no. 4, pp. 538-548, April 2015.

[32] W. Zhou, M. Yang, H. Li, X. Wang, Y. Lin, and Q. Tian, "Towards codebook-free: Scalable cascaded hashing for mobile image search," IEEE Trans. on Multimedia, vol. 16, no. 3, pp. 601-611, April 2014.

[33] D. G. Lowe, "Distinctive image features from scale-invariant keypoints," Int. J. Comput. Vision, vol. 60, no. 2, pp. 91-110, Nov. 2004.

[34] V. Lepetit and P. Fua, "Keypoint recognition using randomized trees," IEEE Transactions on Pattern Analysis and Machine Intelligence, vol. 28, no. 9, pp. 1465-1479, Sept 2006.

[35] E. Rosten and T. Drummond, "Machine learning for high-speed corner detection," in Proceedings of the 9th European Conf. on Computer Vision - Volume Part I, ser. ECCV'06. Springer-Verlag, 2006, pp. 430-443.

[36] M. Agrawal, K. Konolige, and M. Blas, "CenSurE: Center surround extremas for realtime feature detection and matching," in ECCV 2008. Springer Berlin Heidelberg, 2008, vol. 5305, pp. 102-115.

[37] Y. Ke, D. Hoiem, and R. Sukthankar, "Computer vision for music identification," in Computer Vision and Pattern Recognition. IEEE Computer Society Conference on, vol. 1, June 2005, pp. 597-604.

[38] "Text of ISO/IEC CD 15938-13 Compact Descriptors for Visual Search," Tech. Rep., November 2013.

[39] K. Simonyan, A. Vedaldi, and A. Zisserman, "Learning local feature descriptors using convex optimisation," Pattern Analysis and Machine Intelligence, IEEE Trans. on, vol. 36, no. 8, pp. 1573-1585, Aug 2014.

[40] K. Lin, J. Lu, C.-S. Chen, and J. Zhou, "Learning compact binary descriptors with unsupervised deep neural networks," in Computer Vision and Pattern Recognition (CVPR), 2016 IEEE Conference on, 2016, pp. 1183-1192.

[41] Z. Liu, Z. Li, J. Zhang, and L. Liu, "Euclidean and Hamming Embedding for image patch description with convolutional networks," in Proceedings of the IEEE Conference on Computer Vision and Pattern Recognition Workshops, 2016, pp. 72-78.

[42] S. Winder, G. Hua, and M. Brown, "Picking the best daisy," in Computer Vision and Pattern Recognition. IEEE Conf. on, June 2009, pp. 178-185.

[43] H. Jegou, M. Douze, and C. Schmid, "Hamming embedding and weak geometric consistency for large scale image search," in Proceedings of the 10th European Conference on Computer Vision: Part I, ser. ECCV '08. Berlin, Heidelberg: Springer-Verlag, 2008, pp. 304-317.

[44] J. Philbin, O. Chum, M. Isard, J. Sivic, and A. Zisserman, "Object retrieval with large vocabularies and fast spatial matching," in Proceedings of the IEEE Conf. on Computer Vision and Pattern Recognition, 2007.

[45] K. Mikolajczyk, T. Tuytelaars, C. Schmid, A. Zisserman, J. Matas, F. Schaffalitzky, T. Kadir, and L. Gool, "A comparison of affine region detectors," Int. J. of Computer Vision, vol. 65, no. 1-2, pp. 43-72, 2005.

[46] K. Mikolajczyk and C. Schmid, "A performance evaluation of local descriptors," IEEE Trans. Pattern Anal. Mach. Intell., vol. 27, no. 10, pp. 1615-1630, Oct. 2005.

[47] A. Canclini, M. Cesana, A. Redondi, M. Tagliasacchi, J. Ascenso, and R. Cilla, "Evaluation of low-complexity visual feature detectors and descriptors," in Digital Signal Processing (DSP), 2013 18th International Conference on, July 2013, pp. 1-7.

[48] T. S. H. Shao and L. V. Gool, "Zubud-zurich buildings database for image based recognition," Swiss Federal Institute of Technology, Tech. Rep. 260, 2004.

[49] "Cturin180 dataset," http://pacific.tilab.com/www/datasets/, accessed: 2015-05-19.

[50] O. Miksik and K. Mikolajczyk, "Evaluation of local detectors and descriptors for fast feature matching," in Pattern Recognition (ICPR), 2012 21st International Conference on, Nov 2012, pp. 2681-2684.

[51] J. Heinly, E. Dunn, and J.-M. Frahm, "Comparative evaluation of binary features," in Computer Vision - ECCV 2012. Springer Berlin Heidelberg, 2012, pp. 759-773.

[52] J. Figat, T. Kornuta, and W. Kasprzak, "Performance evaluation of binary descriptors of local features," in Computer Vision and Graphics. Springer International Publishing, 2014, vol. 8671, pp. 187-194.
[53] D. Bekele, M. Teutsch, and T. Schuchert, "Evaluation of binary keypoint descriptors," in Image Processing (ICIP), 2013 20th IEEE International Conference on, Sept 2013, pp. 3652-3656.

[54] V. R. Chandrasekhar, D. M. Chen, S. S. Tsai, N.-M. Cheung, H. Chen, G. Takacs, Y. Reznik, R. Vedantham, R. Grzeszczuk, J. Bach, and B. Girod, "The Stanford mobile visual search data set," in Proceedings of the Second Annual ACM Conference on Multimedia Systems, ser. MMSys '11. New York, NY, USA: ACM, 2011, pp. 117-122.

[55] L. Eikvil and M. Holden, "Evaluation of binary descriptors for fast and fully automatic identification," in Pattern Recognition (ICPR), 2014 22nd International Conference on, Aug 2014, pp. 154-159.

[56] S. Arya, D. M. Mount, N. S. Netanyahu, R. Silverman, and A. Y. Wu, "An optimal algorithm for approximate nearest neighbor searching fixed dimensions," J. ACM, vol. 45, no. 6, pp. 891-923, Nov. 1998.

[57] Q. Lv, W. Josephson, Z. Wang, M. Charikar, and K. Li, "Multi-probe LSH: Efficient indexing for high-dimensional similarity search," in Proceedings of the 33rd International Conference on Very Large Data Bases, ser. VLDB '07. VLDB Endowment, 2007, pp. 950-961.

[58] M. Fischler and R. Bolles, "Random sample consensus: A paradigm for model fitting with applications to image analysis and automated cartography," Comm. ACM, vol. 24, no. 6, pp. 381-395, Jun. 1981.

[59] B. Fan, F. Wu, and Z. Hu, "Rotationally invariant descriptors using intensity order pooling," Pattern Analysis and Machine Intelligence, IEEE Transactions on, vol. 34, no. 10, pp. 2031-2045, Oct 2012.

[60] G. Bradski, Dr. Dobb's Journal of Software Tools.

[61] A. Vedaldi and B. Fulkerson, "Vlfeat: An open and portable library of computer vision algorithms," in Proceedings of the International Conf. on Multimedia. New York, NY, USA: ACM, 2010, pp. 1469-1472.

[62] S. Madeo and M. Bober, "Surrey Mobile Dataset," 2016. [Online]. Available: http://cvssp.org/data/surreymobile/

[63] K. Mikolajczyk and C. Schmid, "Scale \& affine invariant interest point detectors," Int. J. of Computer Vision, vol. 60, no. 1, pp. 63-86, 2004.

[64] X. Xu, L. Tian, J. Feng, and J. Zhou, "OSRI: A rotationally invariant binary descriptor," Image Processing, IEEE Transactions on, vol. 23, no. 7, pp. 2983-2995, July 2014

[65] F. Fleuret and I. Guyon, "Fast binary feature selection with conditional mutual information," Journal of Machine Learning Research, vol. 5, pp. $1531-1555,2004$

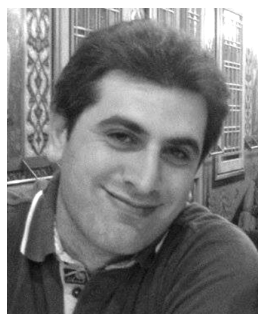

Simone Madeo received the M.S. degree in Computer Engineering in 2011 from University of Pisa, Italy. $\mathrm{He}$ is currently a $\mathrm{PhD}$ candidate at Scuola Superiore Sant'Anna, Pisa, Italy and visiting student at Centre for Vision, Speech and Signal Processing (CVSSP) at University of Surrey, Guildford, UK. His research activity has specific focus on Wireless Sensor Networks applied to multimedia streaming and embedded signal processing for low-complexity visual search and image features extraction.

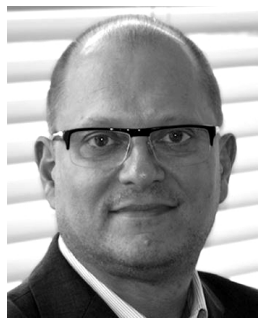

Miroslaw Bober is a Professor of Video Processing at the University of Surrey, U.K. Between 1997 and 2011 he headed Mitsubishi Electric Corporate R\&D Center Europe (MERCE-UK). Prof. Bober received the MSc and PhD degrees from University of Surrey, in 1991 and 1995, respectively. His research interests include various aspects of computer vision and machine intelligence, with recent focus on image/video database retrieval and data mining. He has been actively involved in the development of MPEG standards for over 20 years, chairing the MPEG-7, CDVS and CVDA groups. Prof. Bober is an inventor of over 70 patents and several of his inventions are deployed in consumer and professional products. His publication record includes over 80 refereed publications, including three books and book chapters. 